
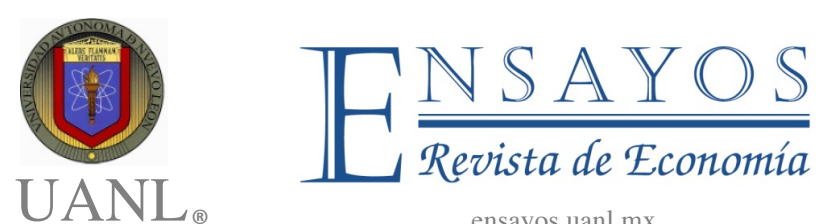

ensayos.uanl.mx

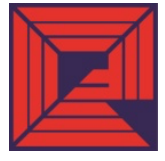

Facultad de Economía

\title{
Los precios del petróleo y la actividad económica en México
}

Oil Prices and Economic Activity in Mexico

\author{
Antonio Ruiz-Porras*§ \\ Javier Emmanuel Anguiano-Pita*
}

\begin{tabular}{|c|c|}
\hline $\begin{array}{l}\text { Información del } \\
\text { artículo }\end{array}$ & Resumen \\
\hline $\begin{array}{l}\text { Recibido: } \\
4 \text { octubre } 2020\end{array}$ & $\begin{array}{l}\text { Estudiamos la interdependencia entre los precios del } \\
\text { petróleo y la actividad económica, en México, usando un } \\
\text { análisis de spillovers estáticos y dinámicos en los dominios }\end{array}$ \\
\hline $\begin{array}{l}\text { Aceptado: } \\
18 \text { octubre } 2021\end{array}$ & $\begin{array}{l}\text { del tiempo y de la frecuencia. Los principales hallazgos } \\
\text { muestran, que: 1) Las relaciones entre los precios del }\end{array}$ \\
\hline $\begin{array}{l}\text { Palabras clave: } \\
\text { Petróleo; Actividad } \\
\text { económica; Spillovers; } \\
\text { Cambio estructural } \\
\text { endógeno; México }\end{array}$ & $\begin{array}{l}\text { petróleo y la actividad económica han fluctuado en el } \\
\text { tiempo; 2) las variaciones de los precios del petróleo tienen } \\
\text { efectos de corto plazo y sus volatilidades, efectos de largo } \\
\text { plazo sobre la actividad económica; 3) los precios del } \\
\text { petróleo MAYA tienen las mayores interdependencias con } \\
\text { la actividad económica; 4) los spillovers netos más altos } \\
\text { entre los precios del petróleo y la actividad económica } \\
\text { ocurrieron entre abril de } 2009 \text { y junio de } 2012 \text {. El estudio } \\
\text { usa series de variaciones y volatilidades mensuales de los } \\
\text { precios spot del petróleo MAYA, WTI y Brent y del } \\
\text { indicador IGAE, del período de febrero de } 1993 \text { a } \\
\text { diciembre de } 2019 \text {. }\end{array}$ \\
\hline
\end{tabular}

* Universidad de Guadalajara; § starp2000@yahoo.com ISSN Electrónico: 2448-8402 | ISSN Impreso: 1870-221X | (C2021 Los autores @) ()

Citar asi: Ruiz-Porras, A. y Anguiano-Pita, J.E. (2021). Los precios del petróleo y la actividad económica en México. Ensayos Revista de Economía, 40(2), 159-188, DOI:10.29105/ensayos40.2-3 


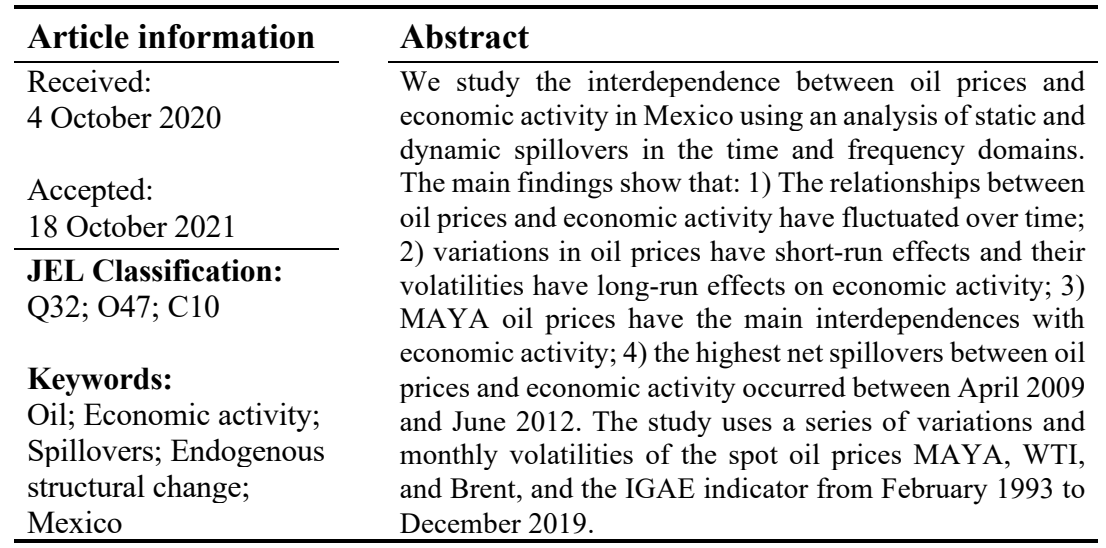

\section{Introducción}

El petróleo y sus derivados proveen energía, insumos y productos esenciales para la economía global. Según la Organización de Países Productores de Petróleo (OPEC, por sus siglas en inglés), el petróleo contabilizó más de 31 por ciento de la demanda global de energía en 2018 (OPEC, 2019: 6). Aún más, según la misma organización, el petróleo contabilizará más del 28 por ciento de la demanda global en el año 2040. La importancia económica del petróleo explica por qué sus precios son constantemente monitoreados, analizados y pronosticados por los agentes económicos y financieros de todo el mundo.

La literatura ha estudiado las relaciones entre los precios del petróleo y las actividades económicas desde los años setenta del siglo pasado (Hamilton, 1983; Brown y Yucel, 2002; Barski y Kilian, 2004). En esta literatura, suele aceptarse que los incrementos y los shocks en los precios del petróleo, tienen efectos negativos sobre la actividad económica. Sin embargo, en muchos países en desarrollo, como México, los precios del petróleo han contribuido a definir las políticas fiscales, los saldos de la balanza comercial y las estrategias de desarrollo. ${ }^{1}$ Aún más, hay quienes señalan que el crecimiento económico de México ha dependido del petróleo (Colmenares, 2008).

La literatura econométrica que estudia las interdependencias entre los precios del petróleo y la actividad económica, suele enfocarse en los países desarrollados que consumen petróleo. Por esta razón, dichos estudios suelen

1 Según la Secretaría de Hacienda y Crédito Público, los ingresos presupuestarios del sector público ascendieron a 22.2 por ciento del PIB, durante 2019 (SHCP, 2020: 57). Asimismo, los ingresos petroleros ascendieron a 3.9 por ciento del PIB. Esto significa que, pese a los problemas financieros del monopolio estatal Petróleos Mexicanos (PEMEX), y la caída en su plataforma de producción registrada en 2019, los ingresos petroleros equivalieron al 17.56 por ciento de los ingresos presupuestarios del sector público de ese año. 
concluir que hay relaciones inversas entre los precios del petróleo y el crecimiento económico (Narayan et. al., 2014; Mohaddes y Pesaran, 2017; Herrera, Karaki y Rangaraju, 2019). Sin embargo, si se considera que los principales productores de petróleo suelen ser países en desarrollo, dicha conclusión es cuestionable. En este contexto, debe mencionarse que los estudios econométricos para países en desarrollo son relativamente escasos.

En este estudio, se analizan las interdependencias entre los precios del petróleo y la actividad económica, en México. El mismo se sustenta en un análisis de spillovers estáticos y dinámicos en los dominios del tiempo y de la frecuencia. La investigación utiliza estadísticas descriptivas, pruebas de cambio estructural endógeno, índices de spillovers estáticos y dinámicos y su descomposición en rangos de frecuencia de corto, mediano y largo plazos. La muestra analizada incluye series de variaciones mensuales y de volatilidades de los precios del petróleo MAYA, WTI y Brent y del indicador IGAE del período comprendido entre febrero de 1993 y diciembre de 2019.

El estudio complementa la literatura empírica sobre las relaciones entre los precios del petróleo y la actividad económica en los países en desarrollo, productores de petróleo. El mismo tiene similitudes metodológicas con los estudios de Blecker (2009), Rodríguez-Benavides y López Herrera (2019) y Zhang et al. (2020). ${ }^{2}$ Sus contribuciones refieren a la caracterización de las mencionadas relaciones en el corto, mediano y largo plazo, considerando precios del petróleo de distintos orígenes y calidades. Particularmente, aquí se utilizan los precios del barril de petróleo de los tipos MAYA, WTI y Brent y el indicador IGAE, como representativos de los precios del petróleo y de la actividad económica.

Las principales diferencias refieren a la naturaleza y periodicidad de las series de tiempo usadas y a la manera de analizar las relaciones de corto y largo plazo. Blecker (2009) y Rodríguez-Benavides y López-Herrera (2019) usan series anuales y trimestrales del PIB, y usan el precio del barril WTI como proxy del precio del petróleo mexicano. Dado que en la investigación aquí planteada se usan series mensuales del IGAE y de los distintos precios de los tipos de petróleo, las series son relativamente más extensas que las utilizadas en los estudios previos. Además, la serie del tipo de petróleo MAYA se usa como representativa del hidrocarburo producido en el país.

2 Zhang et. al. (2020), estudian las interdependencias entre los precios del petróleo, el gas natural, las condiciones de estrés de los mercados financieros y el crecimiento económico de Estados Unidos. Ellos hallan que los shocks se transmiten con mayor velocidad entre las series de volatilidades en comparación con aquellas de los rendimientos. Más aún, ellos muestran que los spillovers del petróleo crudo a la actividad económica son relativamente mayores en comparación con las del gas natural. 
El análisis de las interdependencias entre los precios del petróleo y la actividad económica se sustenta en índices de spillovers, en los dominios del tiempo y de la frecuencia, siguiendo la metodología de Zhang et al. (2020). Particularmente, se emplea el enfoque de Diebold y Yilmaz (2012) para calcular índices de spillovers direccionales estáticos y dinámicos en el dominio del tiempo. Estos índices miden la proporción del impacto de una variable que es atribuible a los shocks en otra. También se emplea el enfoque de Baruník y Křehlík (2018), para descomponer los índices en rangos de frecuencias asociadas con el corto, mediano y largo plazos. ${ }^{3}$

El estudio se organiza en seis secciones. La sección 2 incluye la revisión de la literatura. La sección 3 plantea la metodología de investigación. La sección 4 describe la base de datos e incluye el análisis estadístico y de cambio estructural endógeno de las series. La sección 5 incluye el análisis de interdependencias mediante índices de spillovers en los dominios del tiempo y de la frecuencia para caracterizar las relaciones de corto, mediano y largo plazo entre los precios del petróleo y la actividad económica. Finalmente, la sección 6 sintetiza los hallazgos, discute sus implicaciones y sugiere algunas líneas de investigación para realizar a futuro.

\section{Revisión de la literatura}

Existe una literatura econométrica relativamente extensa que ha estudiado las relaciones entre los precios del petróleo y las actividades económicas. Esta literatura, como ya se ha mencionado, suele centrarse en los países desarrollados (Brown y Yucel, 2002; Kilian, 2008; Mohaddes y Pesaran, 2017). Los estudios pioneros fueron publicados tras el shock de los precios del petróleo ocurrido en 1973 (Barski y Kilian, 2004). ${ }^{4}$ En este contexto, no resulta sorprendente que la mayoría de los estudios concluyan que: los shocks y los incrementos en los precios del petróleo tienen consecuencias negativas sobre las actividades y el crecimiento económico.

Los estudios que se centran en los países desarrollados se clasifican con base en sus líneas de investigación (Brown y Yucel, 2002; Kilian, 2008; Herrera, Karaki y Rangaraju, 2019). La primera línea de investigación estudia los efectos que tienen las variaciones de los precios del petróleo sobre las actividades económicas. La segunda línea estudia los canales mediante los cuales los shocks en los precios se transmiten hacia las actividades económicas.

3 Debe destacarse que la suma de los índices de spillovers en las distintas frecuencias debe ser igual a los resultados de los índices de spillovers en el dominio del tiempo. Por lo tanto, los enfoques en los dominios del tiempo y de la frecuencia resultan ser complementarios entre sí.

4 Los estudios pioneros incluyen aquellos de Pierce y Enzler (1974), Rasche y Tatom (1977), Hamilton (1983) y Ferderer (1996). 
La tercera línea estudia el posible debilitamiento de las relaciones entre los precios del petróleo y la actividad económica. La última línea estudia las respuestas de las políticas monetaria y energética ante los shocks de precios.

La literatura econométrica para los países en desarrollo es mucho menos extensa; sin embargo, hay estudios que confirman que las mencionadas relaciones también son complejas para dichos países. Nasir, et. al. (2018), por ejemplo, muestran que las relaciones son diferentes para los países exportadores e importadores de petróleo; también muestran que los shocks de precios del petróleo pueden tener efectos simétricos o asimétricos sobre la producción, la inflación y la balanza de pagos. Además, hay quienes muestran que hay efectos directos e indirectos asociados a dichas relaciones (PerillaJiménez, 2010; González y Hernández, 2016).

La literatura econométrica para México sobre las mencionadas relaciones es limitada, pese a que el país es un importante productor de petróleo. ${ }^{5}$ Particularmente, Blecker (2009) halla que los precios del petróleo WTI y un conjunto de cambios estructurales determinaron las tasas de crecimiento del PIB entre 1979 y 2007. Rodríguez-Benavides y López-Herrera (2019), por su parte, hallan que el precio del petróleo WTI incidió sobre el crecimiento económico y la formación bruta de capital fijo entre 1983 y 2017. Más aun, sus hallazgos sugieren que la tasa de crecimiento aumenta cuando hay shocks negativos en el precio del petróleo.

Los estudios de Blecker (2009) y Rodríguez-Benavides y López-Herrera (2019) son importantes para analizar las relaciones entre los precios del petróleo y la actividad económica. Sin embargo, debe señalarse que sus trabajos tienen limitaciones. Particularmente, Blecker (2009) analiza la relación de largo plazo, suponiendo cambios estructurales en 1988 y 1994. Rodríguez-Benavides y López-Herrera (2019) analizan la relación de corto plazo usando un modelo GARCH bivariado de tipo BEKK. Además, tal como ya se ha mencionado, ambos estudios usan series relativamente cortas del PIB y usan el petróleo WTI como proxy del petróleo mexicano.

En esta investigación, se utilizan algunos indicadores que subsanan las limitaciones mencionadas. Particularmente, se utilizan los precios spot del barril de petróleo MAYA como representativos del hidrocarburo producido en

5 La literatura econométrica para México suele estudiar las relaciones del precio del petróleo con diversas variables financieras. Entre estos estudios, se incluyen aquellos de De-JesúsGutiérrez, Vergara-González y Díaz-Carreño (2015), Lorenzo-Valdés, Armenta-Fraire y Durán-Vázquez (2016) y Lorenzo-Valdés y Ruiz-Porras (2019). También hay estudios que vinculan los ingresos del petróleo, el consumo y los precios del petróleo con diversas variables económicas. Entre estos estudios, se incluyen aquellos de Reyes-Loya y Blanco (2008), Ramírez-Pascualli y Hall (2013), Ruiz-Porras y Anguiano-Pita (2015) y Gómez, Ciarreta y Zarraga (2018). 
el país y de los principales referentes a nivel internacional (WTI y Brent). Asimismo, se usa el IGAE como proxy de la actividad económica agregada y como insumo para estimar el crecimiento económico. Estas series son relativamente largas porque tienen una periodicidad mensual y porque se recopilan desde hace varios años. Aquí se enfatizan estas características porque las mismas facilitan el análisis econométrico de las series.

La investigación también se justifica desde una perspectiva económica porque, durante el periodo analizado, ocurrieron diversos eventos relevantes para los mercados internacionales de petróleo y los países exportadores de petróleo. Entre estos, se incluyeron: 1) la crisis del efecto Tequila en 1995; 2) la invasión de Irak en 2003; 3) las alzas en los precios del petróleo de 2003 a 2008; 4) la Crisis Financiera Global ocurrida entre 2007 y 2009; 5) la reforma energética en México de 2013 y 2014; 6) la baja en los precios de petróleo entre 2014 y 2016; y, 7) el establecimiento del acuerdo OPEC+ entre 2016 y 2020.

Los eventos relevantes, como los mencionados arriba, pueden inducir cambios estructurales, i.e. quiebres, en las series de los precios del petróleo y la actividad económica. Por esta razón, las fechas de ocurrencia de los cambios estructurales no necesariamente suelen ser conocidas a priori. ${ }^{6}$ Los cambios estructurales, además, generan problemas para evaluar la existencia de relaciones de largo plazo en las series. ${ }^{7}$ Se enfatizan aquí estas consideraciones porque las mismas justifican el uso de las pruebas de cambio estructural endógeno y del análisis de interdependencias usando índices de spillovers en los dominios del tiempo y de la frecuencia.

La metodología empleada aquí para construir los índices de spillovers utiliza series de variaciones y volatilidades mensuales de los precios del petróleo y la actividad económica. Se usan ambos tipos de series porque hay estudios que validan la conveniencia de considerar la existencia de relaciones diferenciadas entre cada momento de las variables ${ }^{8}$. Particularmente, se sigue la propuesta de Zhang et al. (2020), para descomponer los índices de spillovers en

6 Las pruebas de cambio estructural exógeno y endógeno se utilizan para evaluar la existencia de cambios estructurales en las series de datos. Las primeras suponen que las fechas de ocurrencia de los cambios son conocidas; las segundas no hacen este supuesto. Véase Maddala y Kim (1998) para una introducción a las mencionadas pruebas.

7 Tradicionalmente, la existencia de relaciones de largo plazo se evalúa mediante pruebas de cointegración (Johansen, 1988 y 1995); sin embargo, dichas pruebas asumen que las series no manifiestan cambios estructurales. Existen algunos estudios que han propuesto pruebas cuando hay cambios estructurales; particularmente, Johansen, Mosconi y Nielsen (2002) desarrollan pruebas de cointegración considerando la existencia de cambios estructurales. Sin embargo, su análisis supone que las fechas de ocurrencia son conocidas y que hay una tendencia determinística.

8 Véase los estudios de Diebold y Yilmaz (2012), Lovcha y Pérez-Laborda (2020) y Zhang et al. (2020). 
frecuencias de corto, mediano y largo plazo. El análisis de las frecuencias permite develar los mecanismos de propagación de shocks entre las variables en términos de su dirección y persistencia. ${ }^{9}$

La revisión de la literatura contextualiza esta investigación sobre las relaciones de los precios del petróleo y la actividad económica. Si bien se han enfatizado las razones metodológicas, debe señalarse que esta investigación también se justifica por la relativa falta de estudios sobre dichas relaciones, para países en desarrollo exportadores de hidrocarburos. Asimismo, se justifica por la necesidad de complementar la literatura sobre las mencionadas relaciones para México. Aquí, se enfatizan esas consideraciones, ya que motivan la investigación y su enfoque metodológico.

\section{Metodología de investigación}

Esta investigación comprende cuatro tipos de análisis para estudiar las interdependencias entre los precios del petróleo y la actividad económica en México. El primero es el análisis estadístico de las series de rendimientos y volatilidades de los precios del petróleo y la actividad económica. El segundo es el análisis de cambio estructural endógeno. El tercero es el análisis de interdependencias entre los precios del petróleo y la actividad económica en el dominio del tiempo. Finalmente, el cuarto es la descomposición de los spillovers en rangos de frecuencias de corto, mediano y largo plazo. En este contexto, cabe señalar que los mencionados análisis son relativamente independientes y complementarios entre sí.

El análisis estadístico se utiliza para describir las series de precios nominales del petróleo y de la actividad económica. ${ }^{10} \mathrm{El}$ mismo se sustenta en gráficas, estimaciones de estadística descriptiva, pruebas de normalidad Jarque-Bera, raíces unitarias y de efectos ARCH. Particularmente, la estadística descriptiva y las pruebas de normalidad se utilizan para describir las características de las distribuciones de probabilidad de las series. Las pruebas de raíces unitarias se usan para establecer el orden de integración de las series y seleccionar aquellas idóneas para el análisis de interdependencias. Particularmente, aquí se

9 En este contexto, el hallazgo de la predominancia de la transmisión de spillovers en frecuencias altas supone que los mismos se refieren a fluctuaciones de corto plazo. Por el contrario, la predominancia de los mismos en bajas frecuencias supone que estos se relacionan con fluctuaciones de largo plazo, con efectos persistentes en la variable receptora. 10 El uso de series de precios nominales puede ser argumentable en virtud de que el indicador IGAE se define en términos de un año base. Sin embargo, aquí se sigue esta práctica por consistencia con otros estudios. Hamilton (2003), por ejemplo, caracteriza la relación entre los precios del petróleo y la producción real de Estados Unidos usando series nominales del precio del petróleo. Asimismo, el análisis aquí presentado se replicó usando series deflactadas y se obtuvieron resultados cuantitativamente similares. Los mismos se encuentran disponibles si se solicitan a los autores. 
consideran las pruebas ADF propuestas por Dickey y Fuller (1979). Finalmente, las pruebas de efectos $\mathrm{ARCH}$ se basan en la propuesta de Engle (1982), y se usan para determinar la persistencia de la volatilidad en las series analizadas $^{11}$.

El análisis de cambio estructural endógeno utiliza las pruebas propuestas por Perron, Yamamoto y Zhou (2020). Estas pruebas permiten hallar múltiples quiebres, i.e., cambios estructurales en las series de datos. Más aún, permiten identificar las fechas de ocurrencia de los quiebres de forma endógena, i.e., a partir de la información de los mismos datos. Particularmente, las pruebas Perron-Yamamoto-Zhou (PYZ) permiten hallar quiebres individuales y conjuntos en la media y la varianza de una serie. ${ }^{12}$ La hipótesis nula de las pruebas analiza de forma iterativa la existencia de quiebres condicionados a cambios en la media y/o la varianza de las series. ${ }^{13}$

El análisis de interdependencias en los dominios del tiempo y de la frecuencia se utiliza para estudiar las relaciones entre los precios del petróleo y la actividad económica agregada. Particularmente, en ese análisis, se emplea la metodología de Diebold y Yilmaz $(2009,2012)$-en adelante DY - para estimar índices de spillovers en el dominio del tiempo. Los mencionados índices permiten estimar el impacto de los shocks de los precios de petróleo sobre la actividad económica. Además, se emplea la metodología de Baruník y Křehlík (2018) -en adelante BK- para descomponer los índices en rangos de frecuencias asociadas al corto, mediano y largo plazos.

La metodología DY se utiliza para calcular los índices de spillovers totales y direccionales. Esta metodología se sustenta en la estimación de funciones de impulso-respuesta generalizadas (Koop et al., 1996; Pesaran y Shin, 1998). Se usan estas funciones porque, a diferencia de las funciones impulso-respuesta tradicionales, las mismas no dependen del ordenamiento de las variables de los modelos VAR que les dan origen. Particularmente, las mencionadas funciones

11 Particularmente, aquí se emplea esta prueba para validar el uso de modelos Generalizados Autorregresivos de Heterocedasticidad Condicional (GARCH) para la estimación de las series de volatilidades mensuales de los precios del petróleo.

12 Metodológicamente, la mayoría de las pruebas de cambio estructural suponen que los quiebres pueden ocurrir en la media o en la varianza. Sin embargo, ignorar la posibilidad de que dichos quiebres puedan suceder de forma conjunta, puede inducir sesgos potenciales de estimación (Perron y Yamamoto, 2019). Particularmente, las pruebas PYZ usadas aquí consideran la potencial existencia de quiebres individuales en la media o en la varianza y quiebres conjuntos.

13 Las pruebas de cambio estructural endógeno YPZ requieren que las series analizadas sean estrictamente estacionarias. En este contexto, Perron, Yamamoto y Zhou (2020) explicitan los ajustes que deben hacerse a las series cuando las mismas presentan heteroscedasticidad, correlación serial y distribuciones condicionales no normales. 
se utilizan para sustentar el análisis direccional de la transmisión de shocks entre variables. ${ }^{14}$

La metodología BK se emplea para estimar los mismos índices de spillovers usando la descomposición espectral de la varianza de los modelos VAR. La descomposición espectral permite medir hasta qué punto la incertidumbre de una determinada variable en una frecuencia (o rango de frecuencias), se debe a las perturbaciones de las demás variables del $\mathrm{VAR}^{15}$. Los índices de spillovers asociados a diferentes frecuencias permiten estudiar las velocidades de respuesta de la actividad económica ante shocks en los precios del petróleo. Más aún, permiten evaluar si dichos shocks tienen efectos en el corto, mediano y largo plazos.

La estimación de los índices de spillovers totales y direccionales se realiza en tres fases. En la primera fase, se calculan los índices mediante un procedimiento estático que considera todas las observaciones de la muestra. En la segunda fase, se calculan los mismos indicadores de forma dinámica estimando modelos VAR en un contexto de ventanas móviles. Particularmente, cada ventana móvil incluye $N$ subconjuntos de $m$ observaciones temporales ${ }^{16}$. Así, y para cada ventana, se reestiman los índices de spillovers (enfoque de dominio en el tiempo). Finalmente, en la tercera fase, se descomponen los índices empleando la metodología BK.

Metodológicamente, el análisis de los índices de spillovers tiene varias características deseables. El análisis dinámico permite estudiar las fluctuaciones de las interrelaciones entre las variables. Las ventanas móviles, además, reducen los potenciales sesgos en las estimaciones debidos a cambios estructurales. Por otra parte, la descomposición de los índices en rangos de frecuencias permite identificar si las fluctuaciones tienen efectos de corto, mediano y largo plazo en las interrelaciones. Sin embargo, debe mencionarse que el análisis también tiene algunas limitaciones, porque las ventanas y las frecuencias se definen de manera subjetiva.

Finalmente, no sobra señalar que la metodología empleada aquí tiene algunas limitaciones. Las principales son las siguientes: 1) Los valores críticos de las pruebas YPZ pueden ser inadecuados para determinar las fechas de los quiebres si las series manifiestan persistencia estadística; 2) el tamaño de las

14 Para una descripción más detallada, sobre los métodos de cálculo de los índices de spillovers propuestos por la metodología de Diebold y Yilmaz (2012), véase el Apéndice A1.

15 Véase el Apéndice A2, para una descripción detallada del cálculo de los índices de spillovers propuestos por Barunik y Krehlik (2018).

16 El número de ventanas de la muestra, $N$, se define por $N=T-m+1$; donde $T$ es el número total de observaciones de la muestra, mientras que $m$ es el número de observaciones de cada ventana. Particularmente, aquí $T=323, m=48$ y $N=276$. 
ventanas y las frecuencias para calcular los índices de spillovers dinámicos se establece a priori; 3 ) los valores altos y bajos de las magnitudes y variabilidades de los spillovers se establecen de manera relativa; y, 4) los modelos VAR estimados requieren de parámetros definidos de manera relativamente subjetiva. ${ }^{17}$

\section{Base de datos y análisis estadístico}

La base de datos utilizada aquí se integra por series de variaciones y volatilidades mensuales del indicador IGAE y de los precios del petróleo de los tipos MAYA, WTI y Brent. El periodo estudiado abarca de febrero de 1993 a diciembre de 2019. El IGAE utiliza como referencia el año 2013 y se considera ajustado por variaciones estacionales. Por su parte, los precios del petróleo se miden en dólares nominales por barril, sin ajustes estacionales. ${ }^{18}$ La tabla 1 muestra las series de datos y sus fuentes. Las series de variaciones mensuales corresponden a las primeras diferencias de los datos originales en logaritmos, i.e., $r_{t}=\left(\ln P_{t}-\ln P_{t-1}\right) * 100$.

Las series de volatilidades de los precios del petróleo se estimaron a través de la especificación de modelos $\operatorname{ARMA}(p, q)-\operatorname{GARCH}(1,1)$, que pueden representarse mediante las siguientes expresiones:

$$
\begin{aligned}
& r_{t}=\mu+\sum_{i=1}^{p} \phi_{i} r_{t-p}+\sum_{i=1}^{q} \theta_{i} \varepsilon_{t-i}+\varepsilon_{t} \\
& h_{t}=\omega+\alpha h_{t-1}+\beta \varepsilon_{t-1}^{2}
\end{aligned}
$$

donde $r_{t}$ son las series de variaciones mensuales del IGAE y los precios del petróleo y $h_{t}$ es la ecuación que define la varianza condicional. La estimación de la varianza condicional asume una distribución de probabilidad $t$ de Student para las perturbaciones.

17 La estimación de la descomposición de la varianza del error de pronóstico, mediante modelos VAR, requiere la especificación del número de rezagos del sistema y el número de períodos $H$ - adelante del error de pronóstico. Por consistencia con la literatura, en esta investigación, se emplea el criterio de información de Schwarz-Bayesiano (BIC) para seleccionar el orden de rezagos del VAR. Asimismo, siguiendo a Zhang et al. (2020), se considera un horizonte de pronóstico, de 100 períodos hacia adelante.

18 Previo al análisis estadístico se analizó la conveniencia de ajustar la posible estacionalidad de las series originales de precios del petróleo usando el procedimiento X-13 ARIMA. Sin embargo, los resultados no mostraron evidencia significativa de estacionalidad. Por razones de espacio, estos resultados no se presentan aquí, pero se encuentran disponibles si se les solicitan a los autores. 
Tabla 1

Series utilizadas y fuentes de información

\begin{tabular}{|c|c|c|c|}
\hline Variable & Abreviatura & $\begin{array}{c}\text { Unidad } \\
\text { de } \\
\text { medida }\end{array}$ & Fuente \\
\hline $\begin{array}{l}\text { Indicador Global de la Actividad } \\
\text { Económica }\end{array}$ & IGAE & Índice & INEGI \\
\hline Precio spot del tipo de petróleo Maya & MAYA & $\begin{array}{l}\text { USD por } \\
\text { barril }\end{array}$ & EIA \\
\hline $\begin{array}{l}\text { Precio spot del tipo de petróleo Western } \\
\text { Texas Intermediate }\end{array}$ & WTI & $\begin{array}{l}\text { USD por } \\
\text { barril }\end{array}$ & EIA \\
\hline Precio spot del tipo de petróleo Brent & BRENT & $\begin{array}{l}\text { USD por } \\
\text { barril }\end{array}$ & EIA \\
\hline
\end{tabular}

Notas: El indicador IGAE está expresado en términos reales (Base 2013=100) y ajustado estacionalmente. Los precios spot de los tipos de petróleo consideran el costo de importación Free-on-Board (FOB) por barril a Estados Unidos, y se expresan en dólares nominales sin ajustes estacionales. EIA se refiere a la Administración de Información Energética (Energy Information Administracion, por sus siglas en inglés). Las series analizadas comprenden datos mensuales para el período de febrero de 1993 a diciembre de 2019. Fuente: elaboración propia.

Tabla 2

Estadísticas descriptivas de las series de variaciones y volatilidades mensuales

\begin{tabular}{|c|c|c|c|c|c|c|c|c|}
\hline \multirow[t]{2}{*}{ Estadístico } & \multicolumn{4}{|c|}{ Variaciones mensuales } & \multicolumn{4}{|c|}{ Volatilidades } \\
\hline & IGAE & MAYA & WTI & Brent & IGAE & MAYA & WTI & Brent \\
\hline Promedio & 0.1847 & 0.4659 & 0.3549 & 0.4190 & -0.3144 & 2.1725 & 2.0472 & 2.1020 \\
\hline D.E. & 0.8400 & 9.7485 & 8.2333 & 8.6869 & 0.2082 & 0.2425 & 0.1547 & 0.2511 \\
\hline Mínimo & -6.1635 & -42.7143 & -33.1981 & -31.0955 & -0.5307 & 1.7967 & 1.8270 & 1.5795 \\
\hline Máximo & 3.9371 & 33.4472 & 21.3866 & 20.0671 & 0.9427 & 3.0316 & 2.7752 & 2.9136 \\
\hline Sesgo & -1.3236 & -0.7568 & -0.7085 & -0.7336 & 2.6314 & 0.9074 & 1.5306 & 0.5022 \\
\hline Curtosis & 14.8808 & 5.1829 & 4.4466 & 4.1376 & 12.1276 & 3.6196 & 6.0582 & 3.1012 \\
\hline $\begin{array}{l}\text { Jarque- } \\
\text { Bera }\end{array}$ & 1994.008 & 94.9617 & 55.1862 & 46.3851 & 1494.01 & 49.4889 & 251.9963 & 13.7130 \\
\hline $\mathrm{P}$-value & 0.0000 & 0.0000 & 0.0000 & 0.0000 & 0.0000 & 0.0000 & 0.0000 & 0.0065 \\
\hline $\mathrm{ADF}$ & -6.7289 & -7.8291 & -7.3499 & -7.1142 & -4.2665 & -3.8488 & -4.11681 & -3.5168 \\
\hline P-value & 0.0000 & 0.0000 & 0.0000 & 0.0000 & 0.0000 & 0.0169 & 0.0000 & 0.0414 \\
\hline $\mathrm{ARCH}(5)$ & 1490.706 & 185.1192 & 187.8857 & 151.9497 & 0.1153 & 4.0500 & 2.3293 & 2.4640 \\
\hline P-value & 0.0000 & 0.0000 & 0.0000 & 0.0000 & 0.9839 & 0.1695 & 0.4029 & 0.3775 \\
\hline Obs. & 323 & 323 & 323 & 323 & 323 & 323 & 323 & 323 \\
\hline
\end{tabular}

Notas: Las series de variaciones mensuales son aquellas de las primeras diferencias de los valores originales expresados en logaritmos. Las series de volatilidades fueron estimadas mediante un modelo $\operatorname{ARMA}(\mathrm{p}, \mathrm{q})-\mathrm{GARCH}(1,1)$, que considera una distribución de probabilidad condicional $t$ de Student para las perturbaciones. La hipótesis nula de las pruebas de normalidad de Jarque-Bera es que las series se distribuyen normalmente. ADF se refiere a las pruebas de raíz unitaria propuestas por Dickey y Fuller (1979). ARCH(5) se refiere a las pruebas de multiplicadores de Lagrange para efectos $\mathrm{ARCH}$ propuestas por Engle (1982). Dichas pruebas consideran cinco rezagos de cada serie. Las series analizadas comprenden datos mensuales para el período comprendido entre febrero de 1993 a diciembre de 2019. Fuente: elaboración propia con datos de INEGI y EIA.

La tabla 2 muestra las estadísticas descriptivas y las pruebas de estacionariedad de las series de variaciones y volatilidades mensuales. La tabla muestra que las series manifiestan ciertas regularidades: 1) las medias de las series de los precios del petróleo MAYA son más altas que las correspondientes a los tipos de petróleo WTI y Brent; 2) los precios del petróleo son relativamente más 
volátiles que el IGAE; 3 ) todas las series son estacionarias de acuerdo con los resultados de las pruebas ADF; 4) las series de variaciones mensuales presentan altas curtosis y clusters de volatilidad $\left.{ }^{19} ; \mathrm{y}, 5\right)$ no existe evidencia de efectos ARCH en las series de las volatilidades.

La figura 1 muestra las series de variaciones mensuales. Particularmente, la figura muestra que las series de precios del petróleo y del IGAE tuvieron dinámicas similares. Las variaciones mensuales en los precios del petróleo MAYA oscilaron entre -42.71 y $33.44 \%$. Las variaciones de los precios del petróleo WTI oscilaron entre -33.19 y $21.38 \%$. Las variaciones de los precios del petróleo Brent oscilaron entre -31.09 y $20.06 \%$. La figura también sugiere la existencia de dinámicas atípicas en la serie del IGAE alrededor de 1995 y 2009. Además, la figura sugiere la existencia de dinámicas atípicas en las series de los precios del petróleo, alrededor de 2009.

\section{Figura 1}

Series de variaciones mensuales del IGAE y los precios del petróleo (1993-2019)

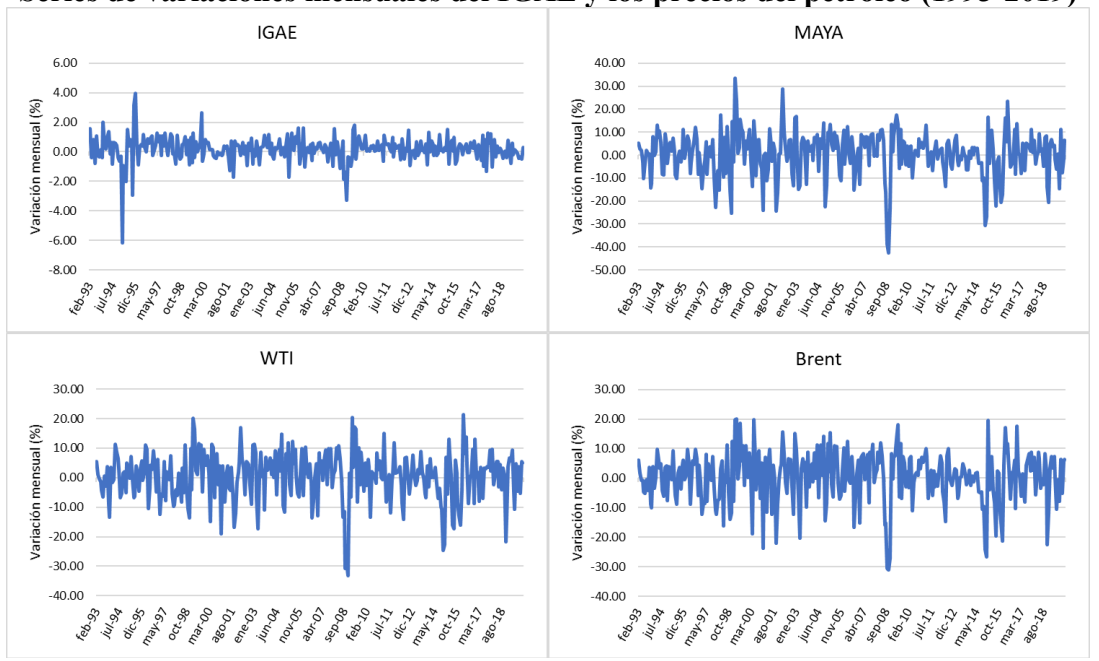

Notas: Las series de variaciones mensuales corresponden a las primeras diferencias de los valores originales en logaritmos. Las series comprenden datos para el período de febrero de 1993 a diciembre de 2019. Fuente: elaboración propia con datos de INEGI y EIA.

La figura 2 muestra las series de las volatilidades mensuales. Las volatilidades del IGAE oscilaron entre 0.58 y $2.56 \%$. Las volatilidades de los precios del petróleo MAYA oscilaron entre 6.02 y $20.73 \%$. Las volatilidades de los precios del petróleo WTI oscilaron entre 6.21 y $16.04 \%$. Las volatilidades de los precios del petróleo Brent oscilaron entre 4.85 y $18.42 \%$. En la figura 2,

19 Los resultados de las pruebas de efectos $\mathrm{ARCH}(5)$ sugieren patrones de persistencia en la volatilidad de las series variaciones mensuales de los precios del petróleo y el IGAE. 
también se confirma la existencia de dinámicas atípicas en la serie del IGAE, alrededor de 1995. Asimismo, en la figura 2, se sugiere la existencia de dinámicas atípicas en las series de los precios del petróleo, alrededor de 2008 у 2009.

La tabla 3 muestra los resultados de las pruebas de cambio estructural endógeno YPZ de las series de variaciones mensuales del IGAE y los precios del petróleo. ${ }^{20}$ La tabla muestra que, de acuerdo con el estadístico $U D M a x L R_{4, T}$, las series de variaciones mensuales del IGAE y del petróleo Brent manifiestan quiebres significativos. La evidencia muestra la ocurrencia de quiebres conjuntos en la media y varianza de la serie del IGAE durante julio de 2009. Asimismo, muestra un quiebre en la varianza en diciembre de 1995. Los quiebres, cabe señalar, se registran durante la Crisis del Efecto Tequila (1994-1995) y la Crisis Financiera Global (2007-2009).

Figura 2

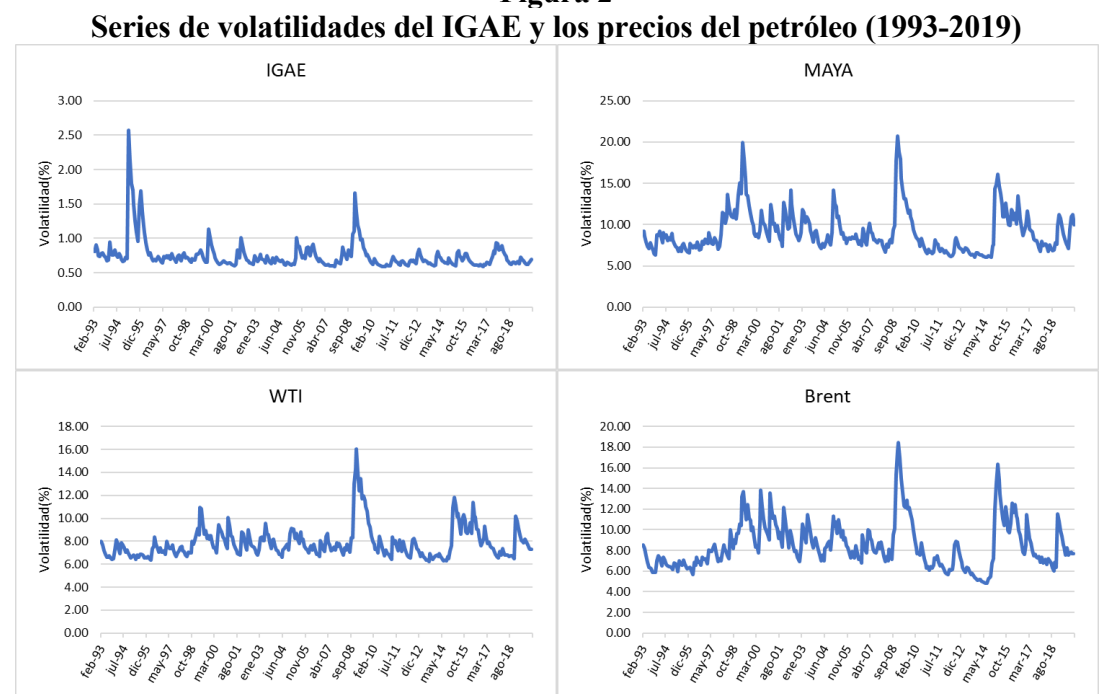

Notas: Las series de volatilidades corresponden a la varianza condicional estimada a partir de modelos $\operatorname{ARMA}(p, q)-\operatorname{GARCH}(1,1)$, que consideran una distribución de probabilidad condicional $t$ de Student para las perturbaciones. Dichas estimaciones consideran, a su vez, las series de variaciones mensuales de las mismas variables. Las series estimadas comprenden datos para el período de febrero de 1993 a diciembre de 2019. Fuente: elaboración propia con datos del INEGI y EIA.

Los principales hallazgos muestran que: 1) las medias de las series de los precios del petróleo MAYA son más altas que las correspondientes a los tipos

20 Los resultados de todos los estadísticos que conforman las pruebas YPZ de cada serie de datos se presentan en el Apéndice A3. 
de petróleo WTI y Brent; 2) los precios del petróleo son más volátiles que el IGAE; 3) todas las series son estacionarias y pueden ser usadas en el análisis de interdependencias; 4) la evidencia muestra cambios estructurales individuales y conjuntos en la media y en la varianza de la serie de variaciones del IGAE y en la media de la serie de variaciones de los precios del petróleo Brent; y, 5) los quiebres de la serie del IGAE se registran durante la Crisis del Efecto Tequila y la Crisis Financiera Global.

Tabla 3

Pruebas de cambio estructural endógeno múltiple en media y varianza YPZ de las series de variaciones mensuales

\begin{tabular}{|c|c|c|c|c|c|}
\hline \multirow[t]{2}{*}{ Variable } & \multirow{2}{*}{$\begin{array}{c}\text { Estadístico } \\
\text { UDmaxLR }_{4, \mathbf{T}}\end{array}$} & \multicolumn{2}{|c|}{$\begin{array}{c}\text { Número óptimo de } \\
\text { quiebres }\end{array}$} & \multirow{2}{*}{$\begin{array}{c}\begin{array}{c}\text { Fechas } \\
\text { de } \\
\text { quiebre } \\
\text { Media }\end{array} \\
\end{array}$} & \multirow[b]{2}{*}{ Varianza } \\
\hline & & Media & Varianza & & \\
\hline IGAE & 9.3511 & 1 & 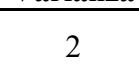 & 2009M07 & $\begin{array}{l}\text { 1995M12; } \\
\text { 2009M07 }\end{array}$ \\
\hline MAYA & 6.1212 & 0 & 0 & & \\
\hline WTI & 5.8945 & 0 & 0 & & \\
\hline Brent & 7.6584 & 1 & 0 & 1997M01 & \\
\hline
\end{tabular}

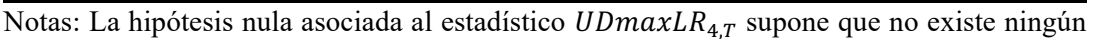
cambio estructural en media y/o varianza. Las pruebas consideran la serie de variaciones mensuales del IGAE y de los precios del petróleo MAYA, WTI y Brent. Las pruebas analizan la existencia de cambios estructurales parciales en un proceso AR (1). Las pruebas suponen que solo pueden ocurrir un máximo de tres quiebres en media y/o varianza. El número de observaciones mínimo de cada segmento analizado se fijó en $\varepsilon=0.10$. Las pruebas consideran la existencia de autocorrelación serial en los términos de error, a partir del uso de estimadores HAC de las matrices de varianza-covarianza siguiendo el método híbrido propuesto por Perron, Yamamoto y Zhou (2020). El número de asteriscos $(*, * *$ y **) se refiere a la significancia de los estadísticos calculados a niveles de 10,5 y 1 por ciento, respectivamente. Las pruebas fueron estimadas en Matlab 2020b, usando los códigos disponibles para descarga gratuita en la página personal en internet de Pierre Perron (http://blogs.bu.edu/perron/files/2020/09/quan200095-sup-0001-dataandprograms.zip). Fuente: Elaboración propia con datos del INEGI y EIA.

\section{Análisis de interdependencias en los dominios del tiempo y de la frecuencia}

En esta sección, se presenta el análisis de interdependencias calculadas en los dominios del tiempo y de la frecuencia para las series de los precios del petróleo y del indicador IGAE. Los índices de spillovers estáticos y dinámicos se calculan usando dos modelos VAR independientes. Particularmente, el primer modelo usa las series de variaciones mensuales; mientras que, el segundo modelo usa las series de volatilidades estimadas mediante los modelos GARCH, usados en la sección previa. Además, las especificaciones de los 
modelos se validan usando pruebas de autocorrelación de residuales y de estabilidad. $^{21}$

El análisis de interdependencias en el dominio de la frecuencia descompone los índices de spillovers en tres diferentes componentes. Siguiendo el enfoque de Zhang et. al. (2020), el primer componente incluye las frecuencias de corto plazo asociadas a períodos de uno a tres meses. El segundo incluye las frecuencias de mediano plazo asociadas a períodos de tres a seis meses. El tercer componente incluye las frecuencias de largo plazo, i.e., mayores a seis meses. Las estimaciones de los índices de spillovers asumen horizontes de pronóstico largos, $H=100$ meses, siguiendo la propuesta de Baruník y Křehlík (2018).

El análisis de interdependencias estático se sustenta en las tablas de conectividad de Diebold y Yilmaz $(2009,2012)$. La diagonal principal de dichas tablas mide los spillovers propios de cada variable. Los elementos fuera de la diagonal principal miden los spillovers cruzados entre las variables. La suma de los elementos de las filas, excluyendo la diagonal principal, muestra los spillovers recibidos de las otras variables en el sistema. De forma análoga, la suma de los elementos de cada columna equivale a la contribución de una variable a las otras contenidas en el sistema. El índice de spillover indicado en negritas se obtiene a partir del promedio de la última fila.

La tabla 4 muestra las tablas de conectividad de las series de los precios del petróleo y del IGAE en el dominio del tiempo. La tabla muestra que el índice de spillover total de las series de variaciones mensuales es mayor $(49.25 \%)$ al de las series de volatilidades (26.96\%). La tabla muestra que la suma de contribuciones de los spillovers de los tipos de petróleo al IGAE asociada a las series de volatilidades (17.51\%) es mayor que la asociada a la suma de las series de variaciones mensuales (7.93\%). Los shocks de los precios del petróleo, por tanto, tienden a transmitirse más rápido al IGAE en términos de volatilidades.

Los spillovers direccionales muestran que las contribuciones de spillovers de los tipos del petróleo al IGAE dependen de las series consideradas en el análisis. Particularmente, las series de variaciones registran que las mayores contribuciones se asocian, de manera decreciente, con los tipos de petróleo WTI $(3.04 \%)$, Brent $(2.89 \%)$ y MAYA $(2.00 \%)$. Las series de volatilidades registran que las mayores contribuciones se asocian con los tipos de petróleo

21 Los modelos usados aquí para calcular los índices de spillovers de las series de variaciones y volatilidades mensuales corresponden a un VAR(3). El orden de rezagos fue seleccionado para evitar problemas de autocorrelación serial. El modelo que usa las series de variaciones mensuales para el análisis estático incluye variables dicotómicas exógenas de impulso para los meses de diciembre de 1995 y julio de 2009 . 
MAYA (10.31\%), WTI (4.99\%) y Brent (2.21\%). Estos hallazgos implican que las actividades económicas no responden de manera uniforme ante los shocks que afectan a los precios del petróleo.

Tabla 4

Índices de spillovers estáticos en el dominio del tiempo

Panel (A): Índices de spillovers de las series de variaciones mensuales.

\begin{tabular}{lccccc}
\hline & IGAE & MAYA & WTI & Brent & De otros \\
\hline IGAE & 92.08 & 2.00 & 3.04 & 2.89 & 7.93 \\
Maya & 0.30 & 38.27 & 30.14 & 31.29 & 61.73 \\
WTI & 0.65 & 30.46 & 36.45 & 32.44 & 63.55 \\
Brent & 0.49 & 31.29 & 31.99 & 36.24 & 63.77 \\
$\begin{array}{l}\text { Contribución a } \\
\text { otros }\end{array}$ & 1.44 & 63.75 & 65.17 & 66.62 & $\mathbf{4 9 . 2 5}$ \\
\hline
\end{tabular}

Panel (B): Índices de spillovers de las series de volatilidades mensuales.

\begin{tabular}{lccccc}
\hline & IGAE & MAYA & WTI & Brent & De otros \\
\hline IGAE & 82.49 & 10.31 & 4.99 & 2.21 & 17.51 \\
MAYA & 1.18 & 64.82 & 19.61 & 14.40 & 35.19 \\
WTI & 0.52 & 6.76 & 73.56 & 19.16 & 26.44 \\
Brent & 0.92 & 9.00 & 18.76 & 71.32 & 28.68 \\
$\begin{array}{l}\text { Contribución a } \\
\text { otros }\end{array}$ & 2.62 & 26.07 & 43.36 & 35.77 & $\mathbf{2 6 . 9 6}$ \\
\hline
\end{tabular}

Notas: La estimación de los índices de spillovers considera el enfoque en el dominio del tiempo propuesto por Diebold y Yilmaz (2012). El modelo VAR subyacente a la estimación de los índices de spillovers de las variaciones mensuales considera una constante y variables dicotómicas de impulso en los meses de diciembre de 1995, enero de 1997 y julio de 2009 como regresores exógenos. Los modelos estimados emplean series de datos mensuales para el período comprendido entre febrero de 1993 y diciembre de 2019. Fuente: elaboración propia con datos de INEGI y EIA.

La tabla 5 muestra los resultados de la descomposición de los índices de spillovers en el dominio de la frecuencia. La tabla muestra que las contribuciones agregadas de spillovers de las series de variaciones y volatilidades de los precios del petróleo al IGAE tienden patrones de comportamiento similares en el tiempo. Así, en ambos casos, las contribuciones crecen entre el corto plazo y el largo plazo tras un ajuste al crecimiento durante el mediano plazo. Además, la tabla muestra que las contribuciones asociadas a las series de variaciones superan a sus contrapartes asociadas a las series de volatilidades en todas las frecuencias consideradas. ${ }^{22}$

22 El Panel A muestra que las contribuciones de spillovers de las series de variaciones asociadas a las frecuencias de corto, mediano y largo plazos ascienden, respectivamente, al $3.24,0.86$ y $3.92 \%$. El Panel B, por su parte, muestra que las contribuciones de spillovers de 
Tabla 5

Índices de spillovers estáticos en el dominio de la frecuencia

Panel (A): Spillovers de las series de variaciones mensuales

Frecuencia: 1 a 3 meses (Corto plazo)

\begin{tabular}{|c|c|c|c|c|c|}
\hline & IGAE & MAYA & WTI & Brent & De otros \\
\hline IGAE & 63.22 & 0.88 & 1.20 & 1.16 & 3.24 \\
\hline MAYA & 0.13 & 18.09 & 14.03 & 14.15 & 28.31 \\
\hline WTI & 0.28 & 13.48 & 17.77 & 14.97 & 28.73 \\
\hline Brent & 0.23 & 15.78 & 16.56 & 19.17 & 32.57 \\
\hline $\begin{array}{l}\text { Contribución a } \\
\text { otros }\end{array}$ & 0.64 & 30.14 & 31.79 & 30.28 & 23.21 \\
\hline \multicolumn{6}{|c|}{ Frecuencia: 3 a 6 meses (Mediano plazo) } \\
\hline & IGAE & MAYA & WTI & Brent & De otros \\
\hline IGAE & 11.10 & 0.20 & 0.32 & 0.34 & 0.86 \\
\hline MAYA & 0.09 & 9.50 & 7.82 & 7.91 & 15.82 \\
\hline WTI & 0.19 & 7.49 & 8.64 & 7.66 & 15.34 \\
\hline Brent & 0.13 & 6.85 & 7.07 & 7.56 & 14.05 \\
\hline $\begin{array}{l}\text { Contribución a } \\
\text { otros }\end{array}$ & 0.41 & 14.54 & 15.21 & 15.91 & 11.52 \\
\hline \multicolumn{6}{|c|}{ Frecuencia:6 meses a infinito (Largo plazo) } \\
\hline & IGAE & MAYA & WTI & Brent & De otros \\
\hline IGAE & 17.67 & 0.96 & 1.54 & 1.42 & 3.92 \\
\hline MAYA & 0.09 & 10.71 & 8.26 & 9.22 & 17.57 \\
\hline WTI & 0.18 & 9.49 & 10.04 & 9.82 & 19.49 \\
\hline Brent & 0.13 & 8.67 & 8.35 & 9.51 & 17.15 \\
\hline $\begin{array}{l}\text { Contribución a } \\
\text { otros }\end{array}$ & 0.40 & 19.12 & 18.15 & 20.46 & 14.53 \\
\hline
\end{tabular}

Panel (B): Spillovers de las series de volatilidades

Frecuencia: 1 a 3 meses (Corto plazo)

\begin{tabular}{|c|c|c|c|c|c|}
\hline & IGAE & MAYA & WTI & Brent & De otros \\
\hline IGAE & 9.04 & 0.12 & 0.41 & 0.37 & 0.90 \\
\hline MAYA & 0.10 & 5.09 & 0.06 & 0.08 & 0.24 \\
\hline WTI & 0.09 & 0.20 & 13.28 & 0.49 & 0.78 \\
\hline Brent & 0.04 & 0.11 & 0.08 & 3.63 & 0.23 \\
\hline Contribución a otros & 0.23 & 0.43 & 0.55 & 0.94 & 0.54 \\
\hline \multicolumn{6}{|c|}{ Frecuencia: 3 a 6 meses (Mediano plazo) } \\
\hline & IGAE & MAYA & WTI & Brent & De otros \\
\hline IGAE & 9.14 & 0.31 & 0.42 & 0.04 & 0.77 \\
\hline MAYA & 0.03 & 4.86 & 0.23 & 0.21 & 0.47 \\
\hline WTI & 0.03 & 0.07 & 11.94 & 0.60 & 0.70 \\
\hline Brent & 0.01 & 0.21 & 0.11 & 3.33 & 0.33 \\
\hline Contribución a otros & 0.07 & 0.59 & 0.76 & 0.85 & 0.57 \\
\hline
\end{tabular}

las series de volatilidades asociadas a las frecuencias de corto, mediano y largo plazos ascienden, respectivamente, al $0.90,0.77$ y $15.83 \%$. 
Frecuencia:6 meses a infinito (Largo plazo)

\begin{tabular}{lccccc}
\hline & IGAE & MAYA & WTI & Brent & De otros \\
\hline IGAE & 64.32 & 9.88 & 4.16 & 1.79 & 15.83 \\
MAYA & 1.05 & 54.87 & 19.31 & 14.10 & 34.46 \\
WTI & 0.39 & 6.49 & 48.34 & 18.07 & 24.95 \\
Brent & 0.88 & 8.68 & 18.57 & 64.36 & 28.13 \\
Contribución a otros & 2.32 & 25.05 & 42.04 & 33.96 & $\mathbf{2 5 . 8 4}$ \\
\hline
\end{tabular}

Notas: La estimación de los índices de spillovers considera el enfoque en el dominio de frecuencias propuesto por Baruník y Křehlík (2018). Los modelos VAR subyacentes a las estimaciones consideran un horizonte de pronóstico $H=100$ meses. Las estimaciones consideran las series de variaciones y volatilidades mensuales para el período comprendido entre febrero de 1993 y diciembre de 2019. Fuente: elaboración propia con datos de INEGI y EIA.

Las mayores contribuciones desagregadas de spillovers de los tipos del petróleo al IGAE dependen del dominio de la frecuencia. Particularmente, las mayores contribuciones en las series de variaciones de corto, mediano y largo plazo corresponden, respectivamente, a los tipos de petróleo WTI (1.20\%), Brent $(0.34 \%)$ y WTI (1.54\%). Las contribuciones en las series de volatilidades corresponden, respectivamente, a los tipos de petróleo WTI $(0.41 \%)$, WTI $(0.42 \%)$ y MAYA (9.88). Estos hallazgos implican que hay distintas velocidades de propagación y de persistencia de los shocks de los precios del petróleo, a la actividad económica.

El análisis estático de las interdependencias entre los precios del petróleo y el IGAE asume que las relaciones son estables en el tiempo. Sin embargo, dicho supuesto es cuestionable por la naturaleza de las variables y el período analizado. Por esta razón, aquí se incluye el análisis de interdependencias dinámico que considera la estimación de los índices de spillovers mediante ventanas móviles de 48 meses $^{23}$. Las ventanas capturan las fluctuaciones cíclicas y los eventos atípicos que podrían alterar las interdependencias. Por complementariedad, el análisis dinámico incluye estimaciones de los índices de spillovers en los dominios del tiempo y de la frecuencia.

La figura 3 muestra las estimaciones de los índices de spillovers total y direccionales netos de las series de variaciones mensuales de los precios del petróleo y el IGAE. El Panel A muestra la dinámica temporal del índice de spillover total. La gráfica muestra que los valores del índice oscilaron entre 48.72\% (marzo de 1998) y 74.99\% (abril de 2009). La descomposición del índice muestra que las contribuciones asociadas a las frecuencias de corto y largo plazos definen en mayor medida al índice de spillover total. Además, la

23 Para probar la robustez de las estimaciones también se calcularon los índices considerando ventanas de 36 y 60 meses y se obtuvieron resultados cuantitativamente similares. Por razones de espacio dichos resultados no se incluyen aquí, pero se encuentran disponibles si se solicitan a los autores. 
figura confirma que las contribuciones asociadas a las frecuencias de largo plazo crecieron, significativamente, durante la Crisis Financiera Global.

Los tres paneles restantes muestran las dinámicas de los spillovers netos entre las series de variaciones mensuales de los precios del petróleo y el IGAE. Las gráficas muestran que el IGAE generalmente es receptor neto de spillover de las variaciones de los precios del petróleo. Particularmente, el valor promedio más alto de dichos índices corresponde al del petróleo MAYA (6.61\%), seguido por el del WTI (6.54\%) y el Brent (5.42\%). Las mismas gráficas también muestran que los índices de spillovers de los tres tipos de petróleo registraron sus valores más altos durante el periodo comprendido entre abril de 2009 y junio de $2012^{24}$.

\section{Figura 3 \\ Índices de spillovers dinámicos de las series de variaciones mensuales de los tipos de petróleo y el IGAE (1997-2019)}

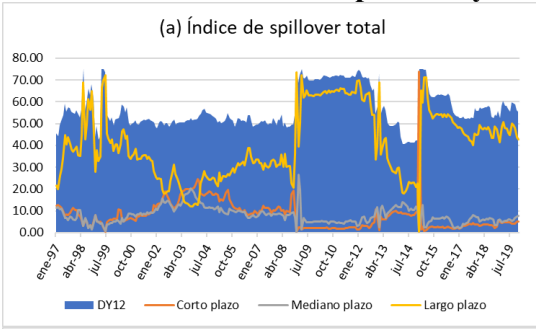

(c) Spillovers direccionales netos IGAE de WTI

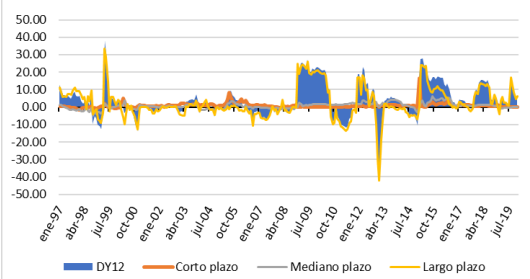

(b) Spillovers direccionales netos IGAE de MAYA

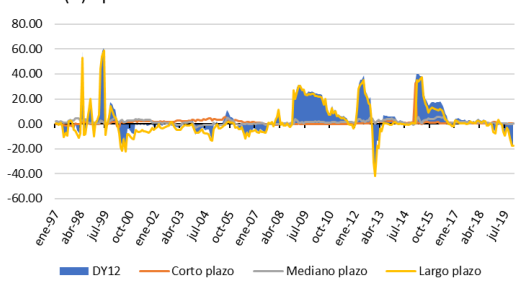

(d) Spillovers direccionales netos IGAE de Brent

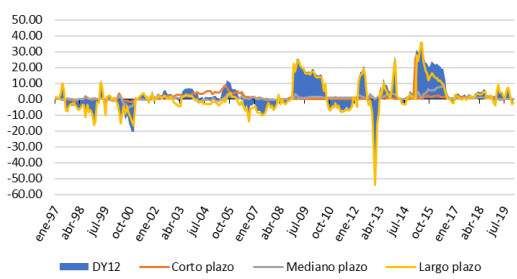

Notas: DY12 se refiere a los índices de spillovers calculados siguiendo la metodología de Diebold y Yilmaz (2012) en el dominio del tiempo. Las bandas de frecuencias de corto, mediano y largo plazo fueron estimadas siguiendo la metodología de Baruník y Křehlík (2018). Las bandas de corto plazo (naranja) corresponde a las frecuencias de 1 a 3 meses. Las bandas de mediano plazo (gris) corresponde a las frecuencias de 3 a 6 meses. Las bandas de largo plazo (amarillo) corresponde a las frecuencias de 6 meses a infinito. Los índices se estiman mediante un modelo VAR (3) con ventanas móviles de 48 meses y un horizonte de pronóstico del error de la varianza $H=100$. Las series de spillovers comprenden

24 Los valores históricos más altos de los índices de spillovers netos de las series de variaciones mensuales entre el IGAE y los tipos de petróleo corresponden al registrado por el WTI (46.29\%) durante abril de 2009, seguido por el del MAYA $(28.44 \%)$ en junio de 2012 y el del Brent (25.46\%) en octubre de 2011. 
observaciones de las variaciones mensuales de los precios del petróleo y el IGAE para el período de enero de 1997 a diciembre de 2019.

La figura 4 muestra las estimaciones de los índices de spillovers total y direccionales netos de las series de volatilidades mensuales de los precios del petróleo y el IGAE. El Panel A muestra el comportamiento del índice de spillover total durante el período analizado. La gráfica muestra que los valores del índice oscilaron entre $40.54 \%$ (abril de 2014) y 75.01\% (febrero de 2015). La descomposición del índice muestra que las contribuciones asociadas a las frecuencias de largo plazo definen en mayor medida al índice de spillover total. Por tanto, la mayor parte de los spillovers en las series de volatilidades ocurren en las frecuencias de largo plazo.

\section{Figura 4 \\ Índices de spillovers dinámicos de las series de volatilidades mensuales de los tipos de petróleo y el IGAE (1997-2019).}

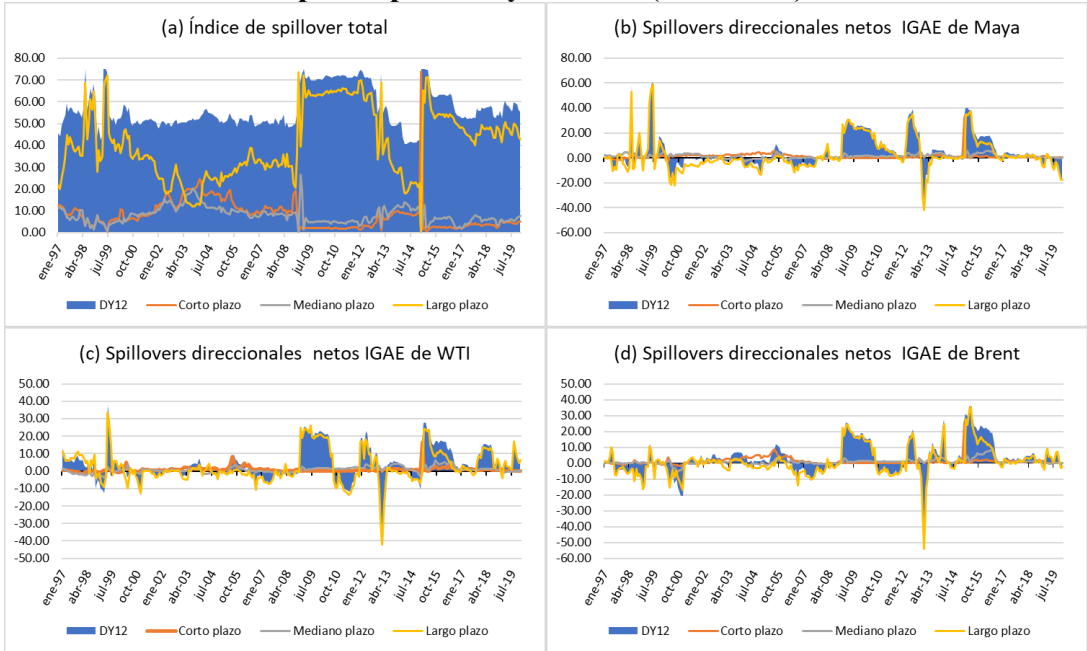

Notas: DY12 se refiere a los índices de spillovers calculados siguiendo la metodología de Diebold y Yilmaz (2012) en el dominio del tiempo. Las bandas de frecuencias de corto, mediano y largo plazo fueron estimadas siguiendo la metodología de Baruník y Křehlík (2018). Las bandas de corto plazo (naranja) corresponde a las frecuencias de 1 a 3 meses. Las bandas de mediano plazo (gris) corresponde a las frecuencias de 3 a 6 meses. La bandas de largo plazo (amarillo) corresponden a las frecuencias de 6 meses a infinito. Los índices se estiman mediante un modelo VAR(3) con ventanas móviles de 48 meses y un horizonte de pronóstico del error de la varianza $H=100$. Las series de spillovers comprenden observaciones de las volatilidades mensuales de los precios del petróleo y el IGAE para el período de enero de 1997 a diciembre de 2019.

Los tres paneles restantes muestran las dinámicas de los spillovers netos entre las series de volatilidades de los precios del petróleo y el IGAE. Las gráficas asociadas a las series de volatilidades tienen una mayor variabilidad que las 
correspondientes a las series de variaciones mensuales; particularmente, los mayores índices promedio de las series de volatilidades corresponden, respectivamente, a los tipos de petróleo MAYA (4.82\%), WTI (3.91\%) y Brent (2.85\%). Las gráficas también muestran que los spillovers crecieron durante la crisis de 2009 y a la caída de los precios del petróleo entre 2014 y 2016.

Los hallazgos del análisis de interdependencias en los dominios del tiempo y de la frecuencia pueden sintetizarse de la siguiente manera: 1) Las relaciones entre los precios del petróleo y la actividad económica en México han fluctuado con el paso del tiempo; 2) las variaciones en los precios del petróleo se asocian con fluctuaciones de corto plazo de la actividad económica; 3 ) la volatilidad de los precios del petróleo se asocia con fluctuaciones de largo plazo de la actividad económica; 4) los precios del petróleo MAYA tienen las mayores interdependencias, i.e., spillovers, con la actividad económica; 5) las mayores interdependencias entre los precios del petróleo y la actividad económica ocurrieron entre los meses de abril de 2009 y junio de 2012.

\section{Conclusiones y discusión}

En este estudio, se han analizado las interdependencias entre los precios del petróleo y la actividad económica en México. El mismo se ha sustentado en un análisis de spillovers estáticos y dinámicos en los dominios del tiempo y de la frecuencia. La investigación ha utilizado estadísticas descriptivas, pruebas de cambio estructural endógeno, índices de spillovers estáticos y dinámicos y su descomposición en rangos de frecuencia de corto, mediano y largo plazos. La muestra analizada ha incluido series de variaciones mensuales y de volatilidades de los precios del petróleo MAYA, WTI y Brent y del indicador IGAE del período comprendido entre febrero de 1993 y diciembre de 2019.

Los principales hallazgos del análisis estadístico muestran que: 1) Las medias de las series de los precios del petróleo MAYA son más altas que las correspondientes a los tipos de petróleo WTI y Brent; 2) los precios del petróleo son más volátiles que el IGAE; 3) todas las series son estacionarias y pueden ser usadas en el análisis de interdependencias; 4) la evidencia muestra cambios estructurales individuales y conjuntos en la media y en la varianza de la serie de variaciones del IGAE y en la media de la serie de variaciones de los precios del petróleo Brent; $\mathrm{y}, 5)$ los quiebres de la serie del IGAE se registran durante la Crisis del Efecto Tequila y la Crisis Financiera Global.

Los hallazgos del análisis de interdependencias en los dominios del tiempo y de la frecuencia pueden sintetizarse de la siguiente manera: 1) Las relaciones entre los precios del petróleo y la actividad económica en México han fluctuado con el paso del tiempo; 2) las variaciones en los precios del petróleo se asocian con fluctuaciones de corto plazo de la actividad económica; 3) la 
volatilidad de los precios del petróleo se asocia con fluctuaciones de largo plazo de la actividad económica; 4) los precios del petróleo MAYA tienen las mayores interdependencias, i.e., spillovers, con la actividad económica; 5) las mayores interdependencias entre los precios del petróleo y la actividad económica ocurrieron entre los meses de abril de 2009 y junio de 2012.

Los hallazgos confirman que las relaciones entre los precios del petróleo y la actividad económica son complejas en los países en desarrollo. Particularmente, los hallazgos sugieren que, a diferencia de la literatura tradicional, los precios del petróleo tienen una relación directa con el crecimiento económico en los países exportadores de petróleo. Asimismo, sugieren que la relación entre los precios del petróleo y la actividad económica pese a sus fluctuaciones tiene una tendencia estable en el largo plazo. Además, los hallazgos sugieren que los shocks en los precios del petróleo pueden transmitirse y tener efectos sobre la actividad económica en el nivel internacional.

Los hallazgos también tienen algunas implicaciones de política económica para México. Las más importantes son las siguientes: 1) Los valores de los índices de spillovers validan que progresivamente está disminuyendo la dependencia del país con respecto a los precios del petróleo y que, por tanto, la economía mexicana se está diversificando; 2) los precios del petróleo MAYA son relativamente más importantes que los del WTI y el Brent para explicar la actividad económica del país; y, 3) la ausencia de cambios estructurales en las series tras la Crisis Financiera Global sugiere que la reforma del sector energético de 2013 no ha tenido efectos de corto ni de largo plazo.

Los hallazgos de esta investigación también validan la pertinencia de desarrollar nuevos estudios sobre las relaciones entre los precios del petróleo con otras variables de las economías en desarrollo. En este contexto, una línea de investigación pertinente es la referida al estudio de las fluctuaciones de las correlaciones entre los precios del petróleo con otras variables de las economías productoras y consumidoras netas de hidrocarburos. Dichos estudios serán útiles para mejorar las políticas energéticas y para promover la estabilidad y el crecimiento de las economías en desarrollo.

\section{Referencias}

[1] Barski, R. B. y Kilian, L. (2004). "Oil and the macroeconomy since the 1970s", Journal of Economic Perspectives, 18(4), 115-134. doi: $10.1257 / 0895330042632708$.

[2] Baruník, J., y Křehlík, T. (2018). "Measuring the frequency dynamics of financial connectedness and systemic risk", Journal of Financial Econometrics, 16(2), 271-296. doi: 10.1093/jjfinec/nby001. 
[3] Blecker, R. A. (2009). "External shocks, structural change, and economic growth in Mexico", 1979-2007", World Development, 37(7), 1274-1284. doi: 10.1016/j.worlddev.2008.10.004.

[4] Brown, S. P. A. y Yucel, M. K. (2002). "Energy prices and aggregate economic activity: An interpretative survey", Quarterly Review of Economics and Finance, 42(2), 193-208. doi: 10.1016/S1062-9769(02)00138-2.

[5] Colmenares, F. (2008). "Petróleo y crecimiento económico en México 1938-2006", EconomíaUnam, 5(15), 53-65.

[6] De-Jesús-Gutiérrez, R., Vergara-González, R. y Díaz-Carreño, M. A. (2015). "Predicción de la volatilidad en el mercado de petróleo mexicano ante la presencia de efectos asimétricos", Lecturas de Economía, 34(65), 299-326. doi: 10.15446/cuad.econ.v34n65.48702.

[7] Diebold, F. X., y Yilmaz, K. (2009). "Measuring financial asset return and volatility spillovers, with application to global equity markets", The Economic Journal, 119(534), 158-171.doi: 10.1111/j.1468-0297.2008.02208.x.

[8] Diebold, F. X., y Yilmaz, K. (2012). "Better to give than to receive: Predictive directional measurement of volatility spillovers", International Journal of Forecasting, 28(1), 57-66. doi: 10.1016/j.ijforecast.2011.02.006.

[9] Dickey, D. A., \& Fuller, W. A. (1979). "Distribution of the estimators for autoregressive time series with a unit root". Journal of the American Statistical Association, 74(366a), 427-431. doi: 10.1080/01621459.1979.10482531.

[10] Engle, R. F. (1982). “Autoregressive conditional heteroscedasticity with estimates of the variance of United Kingdom inflation". Econometrica, 987-1007. doi: $10.2307 / 1912773$.

[11] Ferderer, J. P. (1996). "Oil price volatility and the macroeconomy", Journal of Macroeconomics, 18(1), 1-26. doi: 10.1016/S0164-0704(96)80001-2.

[12] Gómez, M., Ciarreta, A., y Zarraga, A. (2018). "Linear and nonlinear causality between energy consumption and economic growth: The case of Mexico 1965-2014", Energies, 11(4), 784. doi: 10.3390/en11040784.

[13] González, S. y Hernández, E. (2016). "Impactos indirectos de los precios del petróleo en el crecimiento económico colombiano", Lecturas de Economía, (84), 103-141. doi: 10.17533/udea.le.n84a04.

[14] Hamilton, J. D. (1983). "Oil and the macroeconomy since World War II", Journal of Political Economy, 91(2), 228-248. doi: 10.1086/261140.

[15] Hamilton, J. D. (2003). "What is an oil shock?", Journal of Econometrics, 113(2), 363-398: doi: 10.1016/S0304-4076(02)00207-5.

[16] Herrera, A. M., Karaki, M. B., y Rangaraju, S. K. (2019). "Oil price shocks and US economic activity”. Energy Policy, 129, 89-99. doi: 10.1016/j.enpol.2019.02.011.

[17] Johansen, S. (1988). "Statistical analysis of cointegration vectors", Journal of Economic Dynamics and Control, 12(2-3), 231-254. doi: 10.1016/01651889(88)90041-3.

[18] Johansen, S. (1995). Likelihood-based Inference in Cointegrated Vector Autoregressive Models, Nueva York: Oxford University Press.

[19] Kilian, L. (2008). "The economic effects of energy price shocks", Journal of Economic Literature, 46(4), 871-909. doi: 10.1257/jel.46.4.871. 
[20] Koop, G., Pesaran, M. H., y Potter, S. M. (1996). "Impulse response analysis in nonlinear multivariate models". Journal of Econometrics, 74(1), 119-147. doi: 10.1016/0304-4076(95)01753-4.

[21] Lorenzo-Valdés, A. y Ruiz-Porras, A. (2019). "Conditional dependence between oil and exchange rate returns in a developing oil-exporting economy: An investigation with copula-based TGARCH models", International Journal of Global Energy Issues, 42(1/2), 21-44. doi: 10.1504/IJGEI.2019.100686.

[22] Lovcha, Y., y Perez-Laborda, A. (2020). "Dynamic frequency connectedness between oil and natural gas volatilities". Economic Modelling, 84, 181-189. doi: 10.1016/j.econmod.2019.04.008.

[23] Maddala, G.S. y Kim, I. M. (1998). Unit Roots, Cointegration, and Structural Change, Cambridge: Cambridge University Press.

[24] Mohaddes, K., y Pesaran, M. H. (2017). "Oil prices and the global economy: Is it different this time around?", Energy Economics, 65(1), 315-325. doi: 10.1016/j.eneco.2017.05.011.

[25] Narayan, P.K., Sharma, S., Poon, W. C., y Westerlund, J. (2014). "Do oil prices predict economic growth? New global evidence", Energy Economics, 41(1), 137-146. doi: 10.1016/j.eneco.2013.11.003.

[26] Nasir, M. A., Naidoo, L., Shahbaz, M, y Amoo, N. (2018). "Implications of oil prices shocks for the major emerging economies: A comparative analysis of BRICS”, Energy Economics, 76(1), 76-88. doi: 10.1016/j.eneco.2018.09.023.

[27] OPEC, (2019). "World Oil Outlook 2040", Organization of the Petroleum Exporting Countries, Viena: OPEC.

[28] Perilla-Jiménez, J. R. (2010). "El impacto de los precios del petróleo sobre el crecimiento económico en Colombia", Revista de Economía del Rosario, 13(1), 75-116.

[29] Perron, P., y Yamamoto, Y. (2019). "Pitfalls of two-step testing for changes in the error variance and coefficients of a linear regression model". Econometrics, 7(2), 22. doi: 10.3390/econometrics7020022.

[30] Perron, P., Yamamoto, Y., y Zhou, J. (2020). "Testing jointly for structural changes in the error variance and coefficients of a linear regression model". Quantitative Economics, 11(3), 1019-1057. doi: 10.3982/QE1332.

[31] Pesaran, H. H., y Shin, Y. (1998). "Generalized impulse response analysis in linear multivariate models”. Economics Letters, 58(1), 17-29. doi: 10.1016/S01651765(97)00214-0.

[32] Pierce, J. L. y Enzler, J. J. (1974). "The effects of external inflationary shocks", Brookings Papers on Economic Activity, 1974(1), 13-61. doi: 10.2307/2534072.

[33] Ramírez-Pascualli, C. A. y Hall, C. A. S. (2013). "The relation of oil to the Mexican economy: Past, present and future”. En Yáñez-Arancibia, A., Dávalos-Sotelo, R., Day, J.W., y Reyes, E. (Eds.), Ecological Dimensions for Sustainable Socio Economic Development (pp.119-150), Ashurst: WIT Press.

[34] Rasche, R. H. y Tatom, J. A. (1977). "The effects of the new energy regime on economic capacity, production and prices". Federal Reserve Bank of St. Louis Review, 11(5), 2-12.

[35] Reyes-Loya, M. L., y Blanco, L. (2008). "Measuring the importance of oil-related revenues in total fiscal income for Mexico", Energy Economics, 30(5), 25522568. doi: 10.1016/j.eneco.2008.02.001. 
[36] Rodríguez-Benavides, D., y López-Herrera, F. (2019). "Efectos de la incertidumbre de los precios del petróleo en el crecimiento económico de México". Investigación Económica, 78(309), 80-106. doi: 10.22201/fe.01851667p.2019.309.70120.

[37] Ruiz-Porras, A. y Anguiano-Pita, J. E. (2016). "Modelación de las dinámicas, volatilidades e interrelaciones de los rendimientos del petróleo mexicano, BRENT y WTI". Ensayos, Revista de Economía, 35(2), 175-194.

[38] SHCP. (2020). "Documento relativo al cumplimiento de las disposiciones contenidas en el Artículo 42, Fracción I, de la Ley Federal de Presupuesto y Responsabilidad Hacendaria: Pre-criterios 2021". Secretaria de Hacienda y Crédito Público. México: Secretaría de Hacienda y Crédito Público.

[39] Zhang, Y., He, X., Nakajima, T., y Hamori, S. (2020). "Oil, gas, or financial conditions-which one has a stronger link with growth?". The North American Journal of Economics and Finance, 54, 101220. doi: 10.1016/j.najef.2020.101220.

\section{Apéndice A1. Metodología de cálculo de índices de spillovers en el domino del tiempo}

Matemáticamente, la metodología de Diebold y Yilmaz (2012) supone la siguiente representación $M A(\infty)$ de un modelo VAR estacionario en covarianza e invertible:

$Y_{t}=[I-F(L)]^{-1} \xi_{t}=\Lambda(L) \xi_{\mathrm{t}}$

donde $\xi_{t} \sim(0, \Sigma)$ es un vector de innovaciones independiente e idénticamente distribuidas. Los elementos de $\Lambda(L)$ son polinomios de rezagos infinitos que contienen las funciones de impulsorespuesta de las innovaciones posiblemente correlacionadas. Particularmente, Diebold y Yilmaz (2012) proponen emplear el enfoque VAR generalizado de Koop et al. (1996), y Pesaran y Shin (1998), el cual produce una descomposición de la varianza que no depende del orden de las variables en el sistema. De hecho, la misma metodología permite que las innovaciones estén correlacionadas y toma en cuenta este hecho para explicarlas adecuadamente, a través de su distribución histórica observada.

Diebold y Yilmaz (2012) definen la proporción de las innovaciones en la variable $i$ en la varianza del error de pronóstico $H$-pasos adelante de la variable $j$, a partir de la siguiente expresión:

$d_{i j}^{H}=\frac{\sigma_{j j}^{-1} \sum_{h=0}^{H-1}\left(e_{i}^{\prime} \Lambda_{h} \Sigma e_{j}\right)^{2}}{\sum_{h=0}^{H}\left(e_{i}^{\prime} \Lambda_{h} \Sigma \Lambda_{h}^{\prime} e_{i}\right)^{2}}$

donde $\sigma_{j j}$ es la desviación estándar del término de error de la $k$-ésima ecuación del VAR, $\Sigma$ es la matriz de varianzas-covarianzas que contiene las innovaciones del VAR, $e_{i}$ es el vector que permite seleccionar la variable de interés, con 1 como el elemento $i$-ésimo y 0 en otro caso, y $\Lambda_{h}$ es la matriz $h$-rezagada del polinomio $\Lambda(L)$.

En el enfoque VAR generalizado, la suma de las contribuciones propias y cruzadas en la descomposición de la varianza no suman la unidad, i.e. $\sum_{j=1}^{N} d_{i j}^{\sim^{H}} \neq 1$. Por lo tanto, es común que dichas contribuciones sean normalizadas usando la suma de cada fila, i.e. $d_{i j}^{\sim H}=\frac{d_{i j}^{H}}{\sum_{k} d_{i j}^{H}}$, dando como resultado una tabla de contribuciones normalizadas (Diebold y Yilmaz, 2012). ${ }^{25} \mathrm{~A}$ partir de

${ }^{25}$ En general, sucede que la contribución de la variable $j$ a la varianza del error de pronóstico de la variable $i$ no sea exactamente igual, i.e. $C_{i \leftarrow j}^{H} \neq C_{j \leftarrow i}^{H}$. Asimismo, por construcción sucede que $\sum_{j} d_{i j}^{\sim H}=1$ y $\sum_{i, j} d_{i j}^{\sim H}=N$ 
estos ajustes se puede emplear la descomposición de la varianza de un VAR generalizado para construir distintos índices de spillovers.

El índice de spillovers total se puede calcular a partir de la siguiente expresión:

$C^{H}=\frac{1}{N} \sum_{i, j=1, i \neq j}^{N} d_{i j}^{\sim H} * 100$

en donde $0 \leq C^{H} \leq 100$. Este índice mide la contribución de los shocks de volatilidad entre las variables que conforman el VAR a la varianza del error de pronóstico total. En otras palabras, este índice es la suma de las proporciones de la varianza del error de pronóstico de la variable $i$ debido a shocks de la variable $j, \forall i \neq j$.

Adicionalmente, la metodología de Diebold y Yilmaz (2012) permite analizar el componente direccional de los índices de spillovers Particularmente, los spillovers direccionales recibidos por la variable $i$ de todas las otras variables $j, i \neq j\left(C_{i \leftarrow j}^{H}\right)$, y aquellos transmitidos por la variable $i$ a todas las otras variables $j, i \neq j\left(C_{j \leftarrow i}^{H}\right)$, pueden ser calculados mediante las siguientes expresiones:

$C_{i \leftarrow j}^{H}=\sum_{j \neq i}^{N} d_{i j}^{\sim H}$ y $C_{j \leftarrow i}^{H}=\sum_{j \neq i}^{N} d_{j i}^{\sim H}$

Para obtener los índices de spillovers netos simplemente se pueden restar las expresiones presentadas en (A1.4):

$C_{i}^{H}=C_{i \leftarrow j}^{H}-C_{j \leftarrow i}^{H}$

esta medida provee de evidencia con respecto a qué tanto contribuye una de las variables en la volatilidad de las otras. Por último, es posible calcular medidas de spillovers netos por pares de variables para obtener la intensidad de la contribución de la varianza del error de pronóstico entre estas. La medida en cuestión puede calcularse a partir de la siguiente expresión:

$C_{i j}^{H}=d_{i j}^{\sim H}-d_{j i}^{\sim H}$

En general, los índices de spillovers por pares permiten medir la contribución neta resultante de restar los shocks emitidos por la variable $i$ a la variable $j$, menos aquellos recibidos por la variable $i$ de $j$.

\section{Apéndice A2. Metodología de cálculo de índices de spillovers en el dominio de la frecuencia}

Sea $i=\sqrt{-1}$, la representación espectral de la descomposición de la varianza del error de pronóstico (GFEVD) de un modelo VAR en la frecuencia $\omega$ puede ser descrito mediante la siguiente expresión:

$f_{i, j}(\omega)=\frac{\left(\sigma_{j j}^{2}\right)^{-1}\left|\left\{\left[I-F\left(e^{i \omega}\right)\right]^{-1} \Omega\right\}_{i, j}\right|^{2}}{\left\{\left[I-F\left(e^{i \omega}\right)\right]^{-1} \Omega\left[I-F^{\prime}\left(e^{i \omega}\right)\right]^{-1}\right\}_{i i}}$

donde $F\left(e^{i \omega}\right)=\sum_{p} e^{i \omega} F_{p}$ es la transformada de Fourier de las funciones impulso-respuesta de $F$ y $F^{\prime}\left(e^{-i \omega}\right)$ es su transpuesta conjugada compleja.

La expresión (A2.1) representa la proporción de las fluctuaciones de la variable $i$ en la frecuencia $\omega$ debido a shocks en la variable $j$. En la práctica, resulta más útil analizar dichas proporciones en diferentes rangos de frecuencia en lugar de en frecuencias específicas. Por esta razón, Baruník y Křehlík (2018) proponen analizar la GFEVD mediante bandas de frecuencia $d=(a, b): a, b \in$ $(-\pi, \pi), a<b$, las cuales consideran la importancia relativa de las frecuencias que forman cada banda. 
Específicamente, la contribución de un shock a la variable $j$ debido a la variable $i$ en una banda de frecuencias determinada puede ser descrita mediante la siguiente expresión:

$\Theta_{i, j}^{d}=\frac{1}{2 \pi} \int_{a}^{b} P_{i}(\omega) f_{i, j}(\omega) d \omega$

donde $P_{i}(\omega)$ es el espectro de potencia de la variable $i$ en la frecuencia $\omega$ dado por:

$P_{i}(\omega)=\frac{\left[\Lambda\left(e^{i \omega}\right) \Omega \Lambda^{\prime}\left(e^{-i \omega}\right)\right]_{i i}}{\frac{1}{2 \pi} \int_{-\pi}^{\pi}\left[\Lambda\left(e^{i \lambda}\right) \Omega \Lambda^{\prime}\left(e^{-i \lambda}\right)\right]_{i, i} d \lambda}$

De forma similar a la metodología de DY en el dominio del tiempo, las contribuciones mostradas en la expresión (A2.2) son normalizadas de tal forma que $\widetilde{\Theta}_{i, j}^{d}=\Theta_{i, j}^{d} / \sum_{k} \Theta_{i, j}^{\infty}$. En donde $\Theta_{i . j}^{\infty}$ denota la contribución sobre todas las frecuencias, y se ordena posteriormente en una matriz de contribuciones normalizadas $\widetilde{\Theta}^{d}=\left\{\widetilde{\Theta}_{i, j}^{d}\right\}$. Los spillovers al interior de cada banda de frecuencias se definen a partir de las contribuciones normalizadas mediante la siguiente expresión:

$W C^{d}=1-\frac{\operatorname{Tr}\left\{\widetilde{\Theta}^{d}\right\}}{\sum \widetilde{\Theta}^{d}}$

La expresión presentada en la ecuación (A2.4) mide los spillovers que se producen únicamente al interior de cada banda de frecuencias. Esto implica que no tiene en cuenta la importancia de la incertidumbre generada en dicha banda. Así, por ejemplo, si las variables se encuentran fuertemente interrelacionadas en frecuencias altas, pero la proporción de estas es baja respecto a todo el sistema, el índice de spillover total seguirá siendo bajo. Esto último se debe a que las interrelaciones en las frecuencias altas se vuelven insignificante en el agregado.

Baruník y Křehlík (2018) proponen la siguiente expresión para descomponer el índice de spillovers total en diferentes bandas de frecuencia mediante la siguiente expresión:

$F C^{d}=W C^{d} \frac{\sum \widetilde{\Theta}^{d}}{\widetilde{\Theta}^{\infty}}$

la expresión anterior supone que los spillovers al interior de cada banda de frecuencias son ponderados para contabilizar su importancia relativa en el sistema de variables considerado. Los índices de spillovers en el dominio de la frecuencia permiten descomponer los mismos índices obtenidos en el dominio del tiempo en sus componentes asociados a rangos de frecuencia específicos. Sea $H \rightarrow \infty$, el horizonte de pronóstico $H-$ pasos delante de la descomposición de la varianza, y considerando un conjunto de bandas $d_{s} \in D$, que forman una partición del espacio $(-\pi, \pi)$, Baruník y Křehlík (2018) muestran que:

$C=\sum_{d_{s} \in D} F C^{d_{s}}$

Donde $C$ es el índice de spillover total obtenido en el dominio del tiempo de acuerdo con la ecuación (A1.3). Nótese que $H$ no desempeña ningún papel en la expresión anterior para $H \rightarrow \infty$. Sin embargo, de acuerdo con Baruník y Křehlík (2018) la descomposición provee una mejor aproximación para horizontes de pronóstico finitos, siempre que estos no sean demasiado cortos.

La estimación de los índices de spillovers puede ser extendida a medidas direccionales de forma similar a sus versiones obtenidas en el dominio del tiempo. Particularmente, los spillovers direccionales transmitidos de otras variables a $i$, y de $i$ a las otras variables del sistema se definen mediante las siguientes expresiones:

$F C_{i \leftarrow \bullet}^{d}=\left(\sum_{j=1, j \neq i} \widetilde{\Theta}_{i, j}^{d}\right) \frac{\sum \widetilde{\Theta}^{d}}{\sum \widetilde{\Theta}^{\infty}} \mathrm{y} F C_{i \rightarrow \bullet}^{d}=\left(\sum_{j=1, j \neq i} \widetilde{\Theta}_{j, i}^{d}\right) \frac{\sum \widetilde{\widetilde{\Theta}^{d}}}{\sum \widetilde{\Theta}^{\infty}}$

las expresiones presentadas en (A2.7) permiten descomponer los índices de spillovers direccionales obtenidos en el dominio del tiempo a partir de las ecuaciones presentadas en (A1.4) 
en diferentes rangos de frecuencias. Debe señalarse que la suma de los índices en todos los rangos de frecuencias debe ser igual a los índices agregados, obtenidos mediante la metodología de dominio en el tiempo.

\section{Apéndice A3. Pruebas de cambio estructural endógeno en media y varianza de Perron, Yamamoto y Zhou (2020)}

\section{A3.1 Pruebas de cambio estructural en las variaciones mensuales del IGAE}

(a) Pruebas para cambios estructurales en media y/o varianza

\begin{tabular}{|c|c|c|c|c|c|c|c|c|c|c|c|c|c|c|}
\hline \multicolumn{6}{|c|}{ supLR $_{4, T}$} & & \multicolumn{2}{|l|}{$\mathrm{UDmaxLR}_{4, \mathrm{~T}}$} & & & & & & \\
\hline & $m_{a}=1$ & & $m_{\mathrm{a}}=2$ & & $m_{a}=3$ & & $\mathrm{M}=3, \mathrm{~N}=3$ & & & & & & & \\
\hline$n_{a}=1$ & 3.4108 & & 4.2192 & & 7.6584 & ** & 7.6584 & & & & & & & \\
\hline $\mathrm{n}_{\mathrm{a}}=2$ & 2.5629 & & 6.5314 & & 6.9554 & * & & & & & & & & \\
\hline $\mathrm{n}_{\mathrm{a}}=3$ & 5.3421 & & 6.0587 & & 6.2948 & & & & & & & & & \\
\hline \multicolumn{15}{|c|}{ (b) Pruebas para cambios estructurales solo en media } \\
\hline \multicolumn{6}{|c|}{\begin{tabular}{|c|} 
supLR $_{3, \mathrm{~T}}$ \\
\end{tabular}} & & \multicolumn{2}{|l|}{ UDmaxLR $_{3, \mathrm{~T}}$} & \multicolumn{4}{|c|}{ supSeq $_{9, \mathrm{~T}}$} & \multicolumn{2}{|c|}{ Fecha de quiebre } \\
\hline & $m_{a}=1$ & & $m_{a}=2$ & & $\mathrm{~m}_{\mathrm{a}}=3$ & & $M=3$ & & $\mathrm{~m}_{\mathrm{a}}=1$ & $\mathrm{~m}_{\mathrm{a}}=2$ & & $m_{a}=3$ & & \\
\hline $\mathrm{n}_{\mathrm{a}}=0$ & 10.3752 & * & 11.2644 & ** & 10.0074 & ** & 11.2644 & * & 4.0485 & 8.4083 & & 7.0670 & 1997M01 & \\
\hline $\mathrm{n}_{\mathrm{a}}=1$ & 0.23477 & & 0.50553 & & 4.3566 & & 4.3566 & & 4.0485 & 7.7924 & & 4.3786 & & \\
\hline $\mathrm{n}_{\mathrm{a}}=2$ & 0.86161 & & 6.6421 & & 5.5697 & & 6.6421 & & 8.4083 & 6.6063 & & 1.387 & & \\
\hline $\mathrm{n}_{\mathrm{a}}=3$ & 8.1639 & & 5.5899 & & 4.5181 & & 8.1639 & & 7.0453 & 4.1766 & & 1.387 & & \\
\hline \multicolumn{15}{|c|}{ (c) Pruebas para cambios estructurales en varianza } \\
\hline \multicolumn{6}{|c|}{$\operatorname{supLR}_{2, \mathrm{~T}}$} & & \multicolumn{2}{|l|}{ UDmaxLR2 $_{, \mathrm{T}}$} & \multicolumn{4}{|c|}{$\operatorname{supSe} q_{9, \mathrm{~T}}$} & \multicolumn{2}{|c|}{ Fecha de quiebre } \\
\hline & $\mathrm{n}_{\mathrm{a}}=1$ & & $\mathrm{n}_{\mathrm{a}}=2$ & & $\mathrm{n}_{\mathrm{a}}=3$ & & $\mathrm{~N}=3$ & & $\mathrm{n}_{\mathrm{a}}=1$ & $\mathrm{n}_{\mathrm{a}}=2$ & & $\mathrm{n}_{\mathrm{a}}=3$ & & \\
\hline $\mathrm{m}_{\mathrm{a}}=0$ & 6.653 & & 5.9043 & & 6.737 & $*$ & 6.737 & & 3.8143 & 9.9449 & * & 1.5584 & & \\
\hline $\mathrm{m}_{\mathrm{a}}=1$ & 3.0244 & & 4.7198 & & 7.9801 & $* *$ & 7.9801 & & 3.8143 & 9.9449 & & 1.9144 & & \\
\hline $\mathrm{m}_{\mathrm{a}}=2$ & -3.2395 & & 6.6842 & & 8.7569 & $* * *$ & 8.7569 & $*$ & 3.7822 & 16.3917 & *** & 1.9144 & & \\
\hline$m_{a}=3$ & -0.34792 & & 5.2281 & & 7.1856 & $* *$ & 7.1856 & & 3.7496 & 16.3917 & $* * *$ & 3.3756 & & \\
\hline
\end{tabular}

Notas: las pruebas consideran la serie de variaciones mensuales del IGAE. Las pruebas analizan la existencia de cambios estructurales parciales en un proceso $\mathrm{AR}(1)$. Las pruebas suponen que solo pueden ocurrir un máximo de tres quiebres en media y/o varianza. El número de observaciones mínimo de cada segmento analizado se fijó en $\varepsilon=0.10$. Las pruebas consideran la existencia de autocorrelación serial en los términos de error a partir del uso de estimadores HAC de las matrices de varianza-covarianza siguiendo el método híbrido propuesto por Perron, Yamamoto y Zhou (2020). El número de asteriscos $(*, * * y * * *)$ se refiere a la significancia de los estadísticos calculados a niveles de 10,5 y 1 por ciento, respectivamente. Las pruebas fueron estimadas en Matlab 2020b usando los códigos disponibles para descarga gratuita en la página personal en internet de Pierre Perron http://blogs.bu.edu/perron/files/2020/09/quan200095-sup-0001-dataandprograms.zip. $\quad$ Fuente: elaboración propia con datos de INEGI y EIA.

A3.2 Pruebas de cambio estructural en las variaciones mensuales del precio del petróleo MAYA (a) Pruebas para cambios estructurales en media y/o varianza

\begin{tabular}{|c|c|c|c|c|c|c|c|c|c|}
\hline \multicolumn{4}{|c|}{$\operatorname{supLR}_{4, \mathrm{~T}}$} & & $\mathrm{UDmaxLR}_{4, \mathrm{~T}}$ & & & & \\
\hline & $m_{a}=1$ & $m_{a}=2$ & $m_{a}=3$ & & $\mathrm{M}=3, \mathrm{~N}=3$ & & & & \\
\hline$n_{\mathrm{a}}=1$ & 2.2961 & 5.0518 & 6.1212 & & 6.1212 & & & & \\
\hline$n_{a}=2$ & 2.0042 & 4.0505 & 5.5675 & & & & & & \\
\hline$n_{a}=3$ & 2.4948 & 4.1174 & 5.8140 & & & & & & \\
\hline \multicolumn{10}{|c|}{ (b) Pruebas para cambios estructurales solo en media } \\
\hline \multicolumn{4}{|c|}{$\operatorname{supLR}_{3, \mathrm{~T}}$} & & $\mathrm{UDmaxLR}_{3, \mathrm{~T}}$ & \multicolumn{3}{|c|}{ supSeq $_{9, \mathrm{~T}}$} & Fecha de quiebre \\
\hline & $\mathrm{m}_{\mathrm{a}}=1$ & $m_{a}=2$ & $\mathrm{~m}_{\mathrm{a}}=3$ & & $\mathrm{M}=3$ & $\mathrm{~m}_{\mathrm{a}}=1$ & $m_{a}=2$ & $m_{a}=3$ & \\
\hline $\mathrm{n}_{\mathrm{a}}=0$ & 6.2971 & 8.7535 & 7.5531 & & 8.7535 & 1.5268 & 5.9080 & 4.5768 & \\
\hline $\mathrm{n}_{\mathrm{a}}=1$ & 0.0561 & 0.6121 & 1.6045 & & 1.6045 & 1.5268 & 5.6482 & 5.6539 & \\
\hline $\mathrm{n}_{\mathrm{a}}=2$ & 0.9254 & 0.7671 & 1.7681 & & 1.7681 & 5.9080 & 5.9080 & 7.0490 & \\
\hline$n_{a}=3$ & 0.8898 & 1.5892 & 3.0265 & & 3.0265 & 0.9316 & 7.2596 & 0.9198 & \\
\hline \multicolumn{10}{|c|}{ (c) Pruebas para cambios estructurales en varianza } \\
\hline \multicolumn{4}{|c|}{$\operatorname{supLR}_{2, \mathrm{~T}}$} & & UDmaxLR2 $_{, T}$ & \multicolumn{3}{|c|}{ supSeq $q_{9, \mathrm{~T}}$} & Fecha de quiebre \\
\hline & $\mathrm{n}_{\mathrm{a}}=1$ & $\mathrm{n}_{\mathrm{a}}=2$ & $\mathrm{n}_{\mathrm{a}}=3$ & & $\mathrm{~N}=3$ & $\mathrm{n}_{\mathrm{a}}=1$ & $\mathrm{n}_{\mathrm{a}}=2$ & $\mathrm{n}_{\mathrm{a}}=3$ & \\
\hline $\mathrm{m}_{\mathrm{a}}=0$ & 4.5435 & 6.9062 & 7.3314 & ** & 7.3314 & 4.6403 & 8.5717 & 2.1755 & \\
\hline $\mathrm{m}_{\mathrm{a}}=1$ & 2.2783 & 5.9413 & 6.7633 & * & 6.7633 & 4.6403 & 8.7606 & 4.8762 & \\
\hline$m_{a}=2$ & -1.8091 & 5.4942 & 7.3932 & $* *$ & 7.3932 & 4.8339 & 8.7606 & 4.8612 & \\
\hline$m_{a}=3$ & -2.6581 & 5.1495 & 7.8747 & ** & 7.8747 & 5.7747 & 9.9487 & 3.8658 & \\
\hline
\end{tabular}

Notas: las pruebas consideran la serie de variaciones mensuales del precio del petróleo MAYA. Las pruebas analizan la existencia de cambios estructurales parciales en un proceso AR(1). Las pruebas suponen que solo pueden ocurrir un máximo de tres quiebres en media y/o varianza. El número de observaciones mínimo de cada segmento analizado se fijó en $\varepsilon=0.10$. Las pruebas consideran la existencia de autocorrelación serial en los términos de error a partir del uso de estimadores HAC de las matrices de varianza-covarianza siguiendo el método híbrido propuesto por Perron, Yamamoto y Zhou (2020). El número de asteriscos $(* * * \mathrm{y} * * *)$ se refiere a la significancia de los estadísticos calculados a 
niveles de 10,5 y 1 por ciento, respectivamente. Las pruebas fueron estimadas en Matlab 2020b usando los códigos disponibles para descarga gratuita en la página personal en internet de Pierre Perron http://blogs.bu.edu/perron/files/2020/09/quan200095-sup-0001-dataandprograms.zip. elaboración propia con datos de INEGI y EIA.

Fuente:

A3.3 Pruebas de cambio estructural en las variaciones mensuales del precio del petróleo WTI (a) Pruebas para cambios estructurales en media y/o varianza

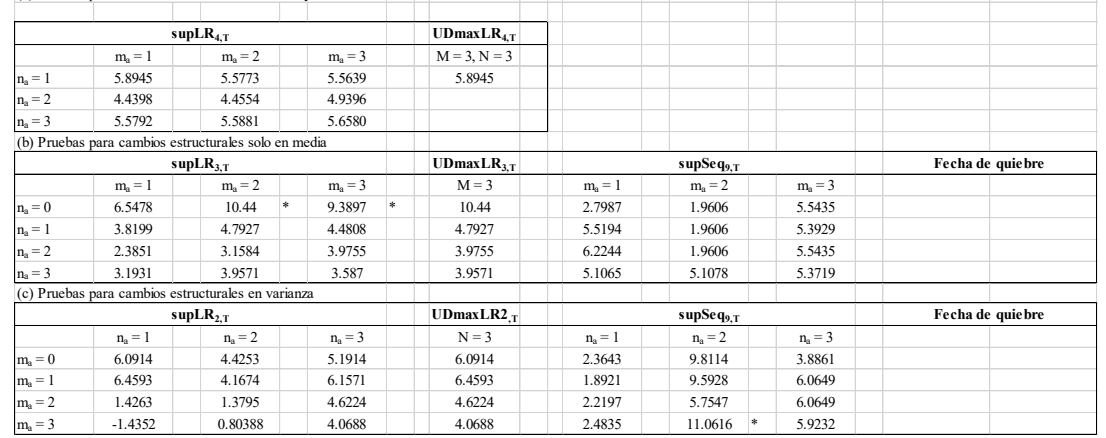

Notas: las pruebas consideran la serie de variaciones mensuales del precio del petróleo WTI Las pruebas analizan la existencia de cambios estructurales parciales en un proceso AR(1). Las pruebas suponen que solo pueden ocurrir un máximo de tres quiebres en media y/o varianza. El número de observaciones mínimo de cada segmento analizado se fijó en $\varepsilon=0.10$. Las pruebas consideran la existencia de autocorrelación serial en los términos de error a partir del uso de estimadores HAC de las matrices de varianza-covarianza siguiendo el método híbrido propuesto por Perron, Yamamoto y Zhou (2020). E1 número de asteriscos $(*, * * \mathrm{y} * * *)$ se refiere a la significancia de los estadísticos calculados a niveles de 10,5 y 1 por ciento, respectivamente. Las pruebas fueron estimadas en Matlab 2020b usando los códigos disponibles para descarga gratuita en la página personal en internet de Pierre Perron http://blogs.bu.edu/perron/files/2020/09/quan200095-sup-0001-dataandprograms.zip.

Fuente: elaboración propia con datos de INEGI y EIA.

A3.4 Pruebas de cambio estructural en las variaciones mensuales del precio del petróleo Brent (a) Pruebas para cambios estructurales en media y/o varianza

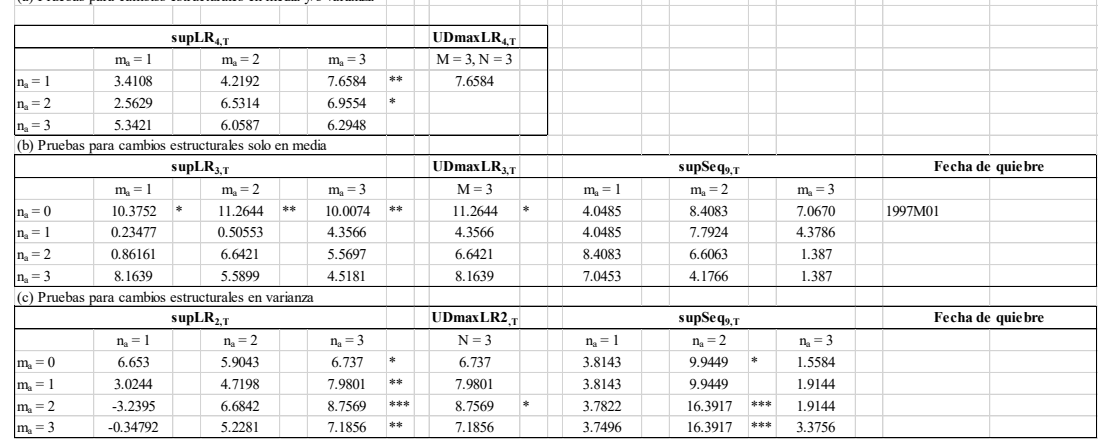

Notas: las pruebas consideran la serie de variaciones mensuales del precio del petróleo Brent. Las pruebas analizan la existencia de cambios estructurales parciales en un proceso AR(1). Las pruebas suponen que solo pueden ocurrir un máximo de tres quiebres en media y/o varianza. El número de observaciones mínimo de cada segmento analizado se fijó en $\varepsilon=0.10$. Las pruebas consideran la existencia de autocorrelación serial en los términos de error a partir del uso de estimadores HAC de las matrices de varianza-covarianza siguiendo el método híbrido propuesto por Perron, Yamamoto y Zhou (2020). El número de asteriscos $(*, * * \mathrm{y} * * *)$ se refiere a la significancia de los estadísticos calculados a niveles de 10,5 y 1 por ciento, respectivamente. Las pruebas fueron estimadas en Matlab 2020b usando los códigos disponibles para descarga gratuita en la página personal en internet de Pierre Perron 
http://blogs.bu.edu/perron/files/2020/09/quan200095-sup-0001-dataandprograms.zip. elaboración propia con datos de INEGI y EIA.

Fuente: 